\title{
LES SOFTWARE FOR THE DESIGN OF LOW EMISSION COMBUSTION SYSTEMS FOR VISION 21 PLANTS
}

\author{
Quarterly Technical Progress Report for
}

October 2001 - December 2001

\author{
by \\ Steve Cannon \\ Baifang Zuo \\ Virgil Adumitroaie \\ Keith McDaniel \\ Cliff Smith
}

January 2002

CFDRC Report No. 8321/5

Contract No.: DE-FC26-00NT40975

submitted to

AAD Document Control, M/S 921-107

National Energy Technology Center

U.S. Department of Energy

P.O. Box 10940

Pittsburgh, PA 15236

Technical Monitor: Mr. Norman T. Holcombe

Contract Monitor: Ms. Crystal Sharp 


\section{DISCLAIMER}

This report was prepared as an account of work sponsored by an agency of the United States Government. Neither the United States Government nor any agency thereof, nor any of their employees, makes any warranty, express or implied, or assumes any legal liability or responsibility for the accuracy, completeness, or usefulness of any information, apparatus, product, or process disclosed, or represents that its use would not infringe privately owned rights. Reference herein to any specific commercial product, process, or service by trade name, trademark, manufacturer, or otherwise does not necessarily constitute or imply its endorsement, recommendation, or favoring by the United States Government or any agency thereof. The views and opinions of authors expressed herein do not necessarily state or reflect those of the United States Government or any agency thereof. 


\begin{abstract}
Further development of a combustion Large Eddy Simulation (LES) code for the design of advanced gaseous combustion systems is described in this fifth quarterly report. CFD Research Corporation (CFDRC) is developing the LES module within the parallel, unstructured solver included in the commercial CFD-ACE+ software. In this quarter, in-situ adaptive tabulation (ISAT) for efficient chemical rate storage and retrieval was further tested in the LES code. The use of multiple trees and periodic tree dumping was investigated. Implementation of the Linear Eddy Model (LEM) for subgrid chemistry was finished for serial applications. Validation of the model on a backstep reacting case was performed. Initial calculations of the SimVal experiment were performed for various barrel lengths, equivalence ratio, combustor shapes, and turbulence models. The effects of these variables on combustion instability was studied. Georgia Tech continues the effort to parameterize the LEM over composition space so that a neural net can be used efficiently in the combustion LES code.

Next quarter, the $2^{\text {nd }}$ consortium meeting will be held at CFDRC. LES software development and testing will continue. Alpha testing of the code will be performed on cases of interest to the industrial consortium. Optimization of subgrid models will be pursued, particularly with the ISAT approach. Also next quarter, the demonstration of the neural net approach, for chemical kinetics speed-up in CFD-ACE+, should be accomplished.
\end{abstract}




\section{TABLE OF CONTENTS}

$\underline{\text { Page }}$

Disclaimer

Abstract

i

List of Figures

List of Tables

1. INTRODUCTION 1

2. EXECUTIVE SUMMARY 1

3. EXPERIMENTAL 1

4. RESULTS AND DISCUSSION 2

4.1 In Situ Adaptive Tabulation (ISAT) 2

4.2 On-Line Linear Eddy Model (LEM) Implementation 5

$\begin{array}{lll}4.3 & \text { LEM/Neural Net Development } & 10\end{array}$

4.4 Premixed SimVal Combustor Case 10

$\begin{array}{ll}\text { 5. CONCLUSION } & 20\end{array}$

$\begin{array}{ll}\text { 6. REFERENCES } & 20\end{array}$

APPENDIX A - WORK SCHEDULE A-1

APPENDIX B - FUTURE PLANS $\quad$ B-1

APPENDIX C - GEORGIA TECH PROGRESS $\quad$ C-1 


\section{LIST OF FIGURES}

Page

Figure 1. Temperature Predictions Using the 1-step, 5-step, and 15-step Chemistry 3

Figure 2. Comparison of Combustor Pressure History Using Direct Integration and ISAT for the 5-step, 9-species Chemistry

Figure 3. Comparisons of Predicted Radial Profiles of NO and OH Using Direct Integration and ISAT with Different Tolerance Levels

Figure 4. OH Contour Predictions at $6 \mathrm{msec}$ Using Direct Integration and ISAT (73\% retrieves)

Figure 5. Schematic Diagram of the Linear Eddy Splicing Events

Figure 6. Computational Domain and Non-reacting Axial Velocity Contours for Backstep Case $(\mathrm{H}=.0254 \mathrm{~m})$

Figure 7. Schlieren Images for LES Predictions and Measurements

Figure 8. $\quad$ Predicted Chemical Reaction Rates

Figure 9. Predicted Axial Velocity Contours Showing Reattachment Lengths using Laminar Chemistry and Linear Eddy Model

Figure 10. SimVal 2D Axisymmetric Geometry for Baseline Case

Figure 11. Steady-state Predictions of Temperature, Axial Velocity, and Eddy Viscosity for Baseline SimVal Case

Figure 12. Time History and Fourier Transformed Signal of Predicted Combustor Pressure at Dome Face for Baseline SimVal Case

Figure 13. Predicted Time Series and Fourier Transform of Combustor Pressure for Case 213

Figure 14. Geometry for Long Pipe Combustor Case

Figure 15. Predicted Time Series and Fourier Transform of Combustor Pressure for Combustor Pipe Case

Figure 16. Predicted Instantaneous Temperature Contours at Max and Min Pressure During Instability Cycle for Straight-pipe and Resonant Section Combustor Cases

Figure 17. Predicted Instantaneous Axial Velocity Contours at Max and Min Pressure During Instability Cycle for Straight-pipe and Resonant Section Combustor Cases

Figure 18. Predicted Instantaneous Heat Release Contours at Max and Min Pressure During Instability Cycle for Straight-pipe and Resonant Section Combustor Cases

Figure 19. Predicted Instantaneous Pressure Contours at Max and Min Pressure During Instability Cycle for Straight-pipe and Resonant Section Combustor Cases

Figure 20. Predicted Instantaneous Temperature Contours at Max and Min Pressure for the Unsteady RANS and LES with LDKM 


\section{LIST OF TABLES}

Page

Table 1. Direct Integration and ISAT Results for Transient Combustor CFD Calculations 3 Table 2. Predicted Oscillations for Varying Barrel Lengths 12

Table 3. Predicted Oscillations for Varying Mixture Equivalence Ratio $\quad 18$

Table 4. $\quad$ Predicted Oscillations for Unsteady RANS and LES 19 


\section{INTRODUCTION}

Vision 21 combustion systems will require innovative low emission designs and low development costs if Vision 21 goals are to be realized. In this three-year project, an advanced computational software tool will be developed for the design of low emission combustion systems required for Vision 21 clean energy plants. The combustion LES software will be able to accurately simulate the highly transient nature of gaseous-fueled turbulent combustion so that innovative concepts can be assessed and developed with fewer high-cost experimental tests. During the first year, the project will include the development and implementation of improved chemistry (reduced GRI mechanism), subgrid turbulence (localized dynamic), and subgrid combustion-turbulence interaction (Linear Eddy and Conditional Moment Closure) models into the CFD-ACE+ code. University expertise (Georgia Tech and UC Berkeley) will be utilized to help develop and implement these advanced submodels in the unstructured, parallel CFD flow solver. Efficient numerical algorithms that rely on in situ look-up tables or artificial neural networks will be used for the expensive subgrid chemical kinetic and mixing calculations. In the second year, the combustion LES software will be evaluated and validated using experimental data from lab-scale and industrial test configurations, including important benchmark data from DOE-NETL. During the last year, seven industrial and academic partners will take the combustion LES code and exercise it on problems of their choice. Final feedback and optimizations will then be implemented in the final release version of the combustion LES software.

\section{EXECUTIVE SUMMARY}

Work in this fifth quarter (October - December 2001) has included the implementation and testing of the Linear Eddy Model in CFD-ACE+ for predicting subgrid chemistry. Also, in situ adaptive tabulation (ISAT) for efficient multi-step chemistry has been further tested and refined. Georgia Tech continues efforts to train a neural net for accurate and efficient chemistry descriptions in the LES software. Initial calculations of the SimVal experiment were carried out to help guide the experiments that will be a database for validating the LES code.

Next quarter, completion of the following tasks are planned:

1. Optimize and refine ISAT for large chemical mechanisms (>19 species).

2. Carry out initial validation of LES code for predicting emissions and instability in cases selected by the industrial consortium.

3. Develop, implement, and test neural net on lean premixed SimVal combustor test case.

\section{EXPERIMENTAL}

No experiments were performed this quarter. 


\section{RESULTS AND DISCUSSION}

\subsection{In Situ Adaptive Tabulation (ISAT)}

In Situ Adaptive Tabulation (ISAT), Pope, 1997, was tested for the methane 5-step (9 species) and 15-step (19 species) reduced mechanisms. Three ISAT methods are available in CFDACE+. ISAT type 1 stores compositions in a table, where the tolerance is a function only of the input composition. First-time compositions are used in the future for queries that are close in composition space. No extrapolation or interpolation is used. ISAT type 2 performs linear extrapolation from existing composition points in the table. ISAT type 2 does not track growth of composition cells in the table, therefore the extrapolation may be inaccurate for large tolerance bands. ISAT type 3 utilizes growth by computing an ellipsoid of accuracy from a Singular Value Decomposition (SVD). ISAT type 3 was used in the test cases shown here. The test cases included the new DOE-NETL perfectly premixed combustor (Maloney, 2001) and the old DOE-NETL partially premixed combustor (Richards and Janus, 1997). Comparisons of accuracy and cpu time between direct integration and ISAT were made. In addition, calculations were performed with staggered chemistry and non-staggered chemistry. The staggered chemistry approach computes integrated reaction rates only once per time step, while the nonstaggered approach computes rates every timestep within the iteration. The transient calculations required 8 iterations per timestep for the premixed combustor case.

The reduced mechanisms, with steady-state species, can only be handled with the operatorsplitting solution technique. Operator splitting only works for transient runs and uses a stiff ODE solver to solve the coupled set of species chemical kinetic equations. An average reaction rate (over the global time step) is then used in the overall conservation equation for each species mass fraction. For an initial steady-state condition, 1-step chemistry without operator splitting is used. Then, the mechanism is switched to either the 5-, 15-, or 16-step mechanisms and the transient run is initiated. An overall timestep of 1e-5 seconds was used for the premixed combustor case. Figure 1 shows the steady-temperature location using the 1-step chemistry and the 5- and 15-step chemistry after 100 timesteps. These results show that all three mechanisms predict a different flame location. This is somewhat surprising for the 5- and 15-step mechanisms since they were both derived from the full GRI mechanism. Further work is required to understand why the 5- and 15-step mechanisms give different predicted flameshapes. The shorter flamezone predicted by the 5-step mechanism allows instability to develop, while the longer flame does not. The weaker coupling for the longer flame is due to a greater distribution in the heat release zone.

ISAT results for the various different mechanisms are presented in Table 1. The ISAT results were obtained by setting the maximum tree records to 20,000 for the 5-step mechanism and 40,000 for the 19-step mechanism. When the maximum tree records was reached, then the tree was dumped and reinitiated from scratch. The results show that a speedup can be achieved with the 5-step, 9 species mechanism. The computational times are reduced by a factor of 2.3 for the staggered chemistry case. Compared to the direct integration/non-staggered case, the ISAT/nonstaggered speedup is 4.4 . 


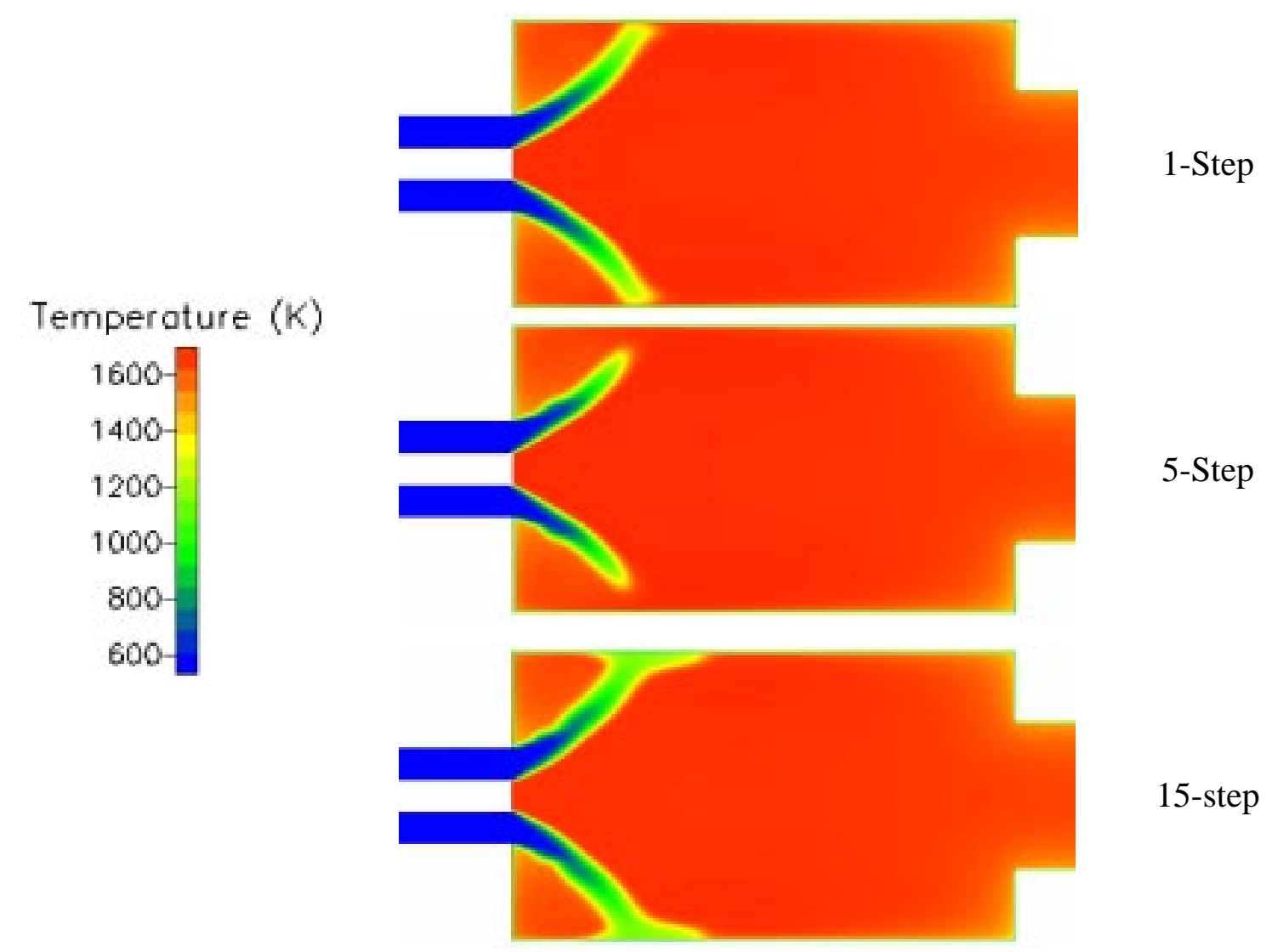

Figure 1. Temperature Predictions Using the 1-step, 5-step, and 15-step Chemistry

Table 1. Direct Integration and ISAT Results for Transient Combustor CFD Calculations

\begin{tabular}{|c|c|c|c|c|}
\hline $\begin{array}{c}\text { Premixed Case } \\
\text { Mechanism }\end{array}$ & $\begin{array}{l}\text { Kinetics } \\
\text { Solution }\end{array}$ & Error Tolerance & \% Retrieves & CPU Time \\
\hline 5-step & $\begin{array}{c}\text { DI/Non- } \\
\text { Staggered } \\
\end{array}$ & 一 & - & $400 \mathrm{~min}$. \\
\hline 5-step & DI/Staggered & - & - & 39 min. \\
\hline 5-step & $\begin{array}{l}\text { ISAT/Non- } \\
\text { Staggered }\end{array}$ & $1 e-4$ & 88 & $91 \mathrm{~min}$. \\
\hline 5-step & ISAT/ Staggered & $1 \mathrm{e}-4$ & 82 & $17 \mathrm{~min}$. \\
\hline 5-step & ISAT/ Staggered & $\begin{array}{l}\text { 1e-4 (tighter } \\
\text { radicals) }\end{array}$ & 73 & $22 \mathrm{~min}$. \\
\hline 15-step & DI/Staggered & - & - & $45 \mathrm{~min}$. \\
\hline 15-step & ISAT/ Staggered & $1 \mathrm{e}-4$ & 35 & $66 \mathrm{~min}$. \\
\hline \multicolumn{5}{|c|}{ Partially Premixed Case } \\
\hline 5-step & DI/Staggered & - & - & $45 \mathrm{~min}$. \\
\hline 5-step & ISAT/ Staggered & $1 \mathrm{e}-4$ & 70 & $32 \mathrm{~min}$. \\
\hline
\end{tabular}


Figure 2 shows predicted transient pressure results with ISAT and direct integration. Good agreement is found when using an ISAT tolerance of $1 \mathrm{e}-4$ (82\% retrieves). Figure 3 shows predicted radial profiles of $\mathrm{OH}$, and $\mathrm{NO}$ mass fraction using direct integration and ISAT with various tolerance levels. The tighter tolerance ( $73 \%$ retrieves) ISAT was needed to predict the $\mathrm{OH}$ and $\mathrm{NO}$ concentrations in the central recirculation zone. It appears that long residence time fluid is more sensitive to the ISAT tolerance. Comparisons of major species and temperature were excellent between direct integration and ISAT for either tolerance level. Figure 4 shows the entire flame zone comparisons between direct integration and the $73 \%$ retrieve ISAT. Good agreement was obtained.

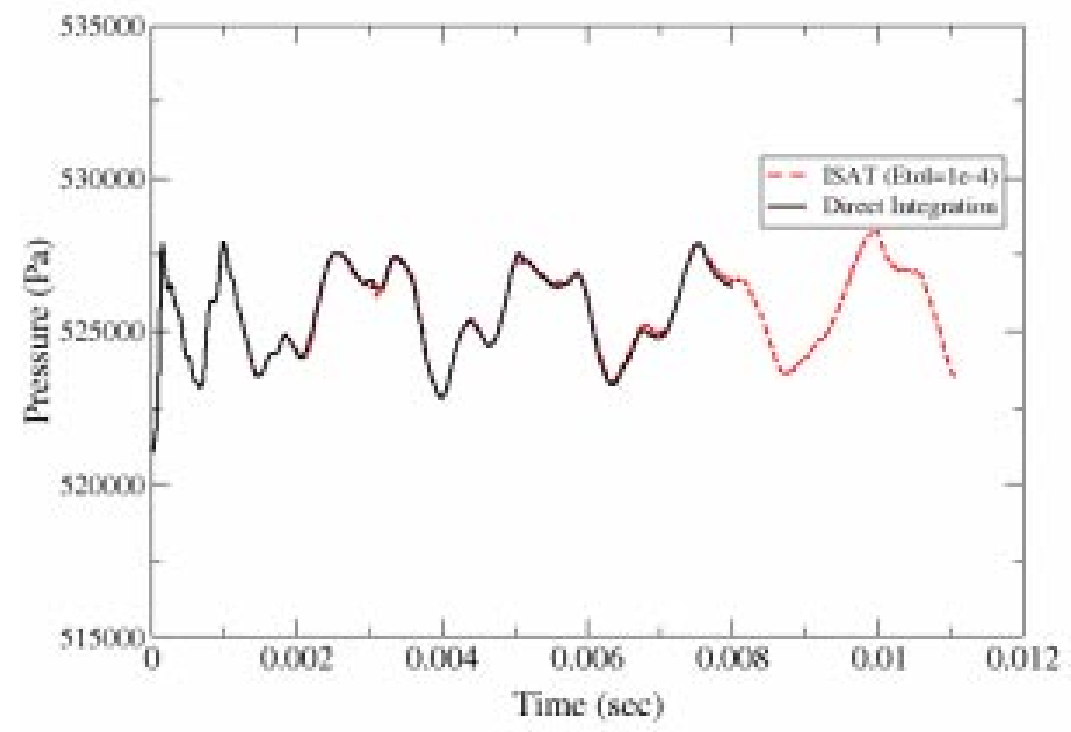

Figure 2. Comparison of Combustor Pressure History Using Direct Integration and ISAT for the 5-step, 9-species Chemistry
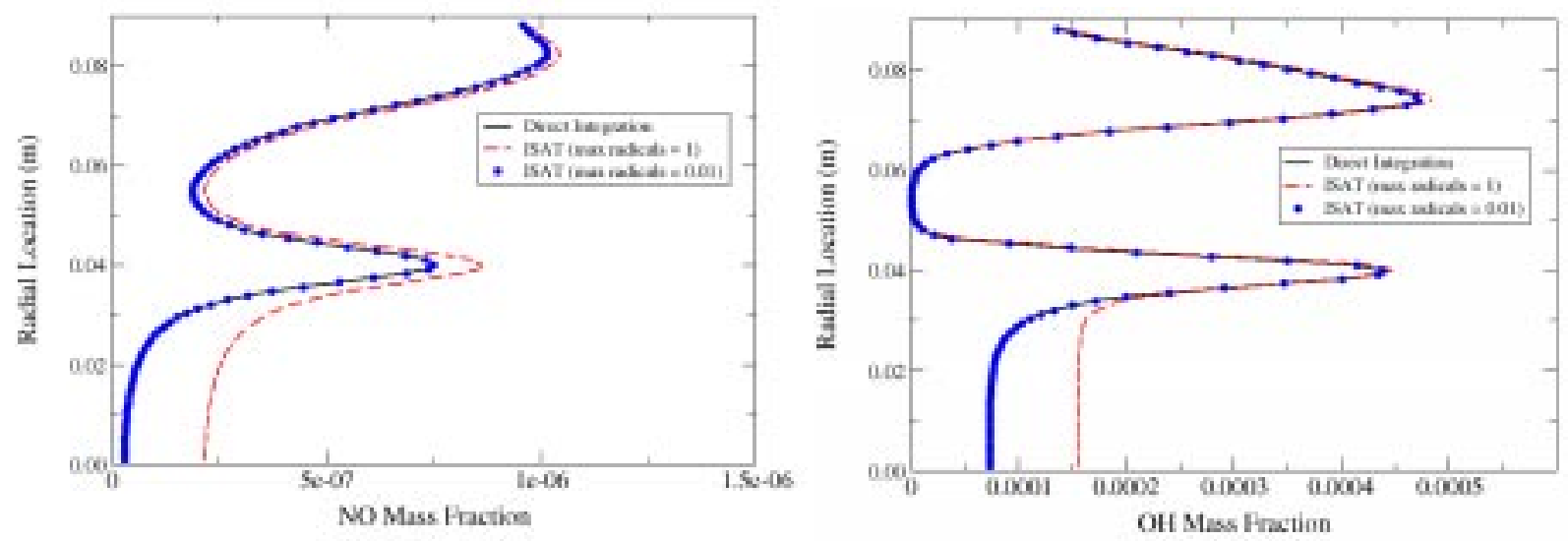

Figure 3. Comparisons of Predicted Radial Profiles of $\mathrm{NO}$ and $\mathrm{OH}$ Using Direct Integration and ISAT with Different Tolerance Levels 


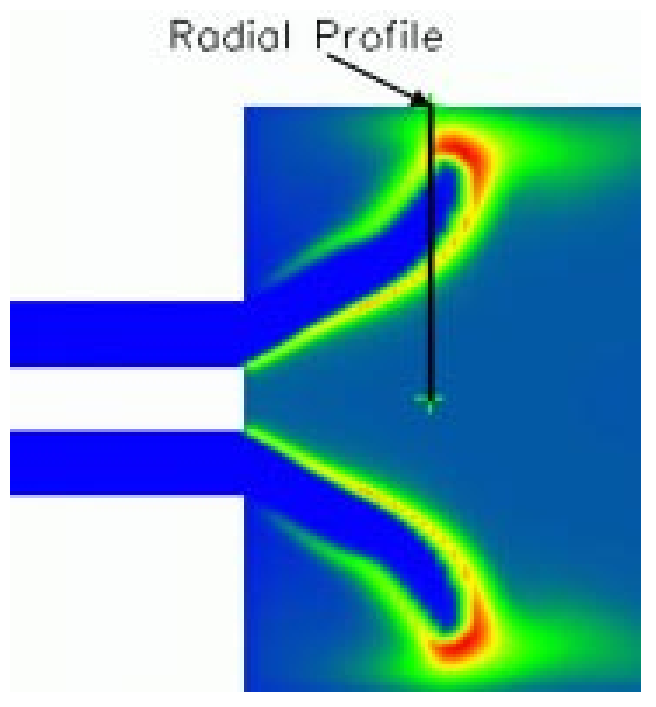

Direct Integration

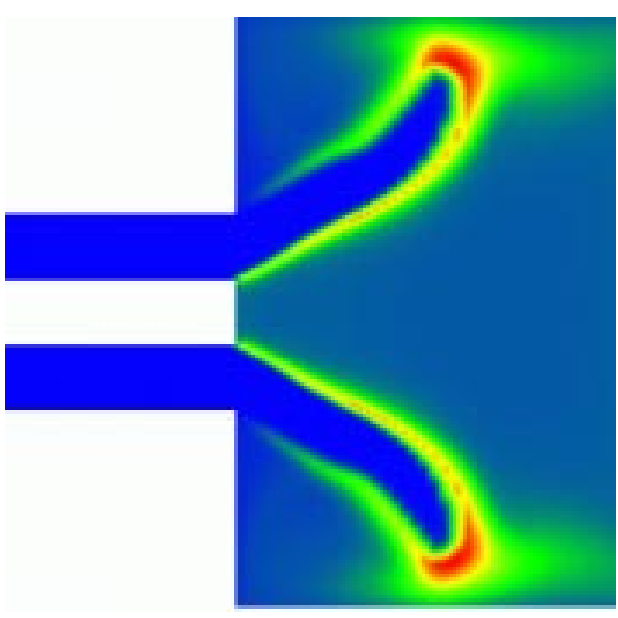

ISAT

Figure 4. OH Contour Predictions at 6 msec Using Direct Integration and ISAT (73\% retrieves)

For the 15-step chemistry, a speed-up was not achieved with ISAT, even though $35 \%$ of the computations were retrievals. These results show that for more detailed chemistry, the retrieval process is fairly expensive - even more than direct integration. Pope and coworkers have reported speedups of 10-1000 for Monte Carlo PDF calculations. To achieve this speed-up, $99 \%$ of the chemical kinetic calculations would need to be fast retrievals. Approaches for improving the ISAT method are being investigated, including: multiple tree storage, more accurate mapping gradient solution, more accurate and/or efficient singular value decomposition, and a more efficient tree structure (PK-tree). These new improvements in ISAT will be needed to do efficient LES calculations with the 15 -step mechanism.

\subsection{On-Line Linear Eddy Model (LEM) Implementation}

\subsubsection{Model Description}

The Linear Eddy Model (LEM) for describing subgrid chemistry was implemented and tested in the unstructured CFD-ACE+ flow solver. The LEM describes the effects of turbulent stirring, molecular diffusion, and chemical reaction at scales down to the molecular level where reaction ultimately occurs. The LEM achieves this by subdividing each LES cell into a smaller onedimensional domain. Along the one-dimensional domain, molecular diffusion and chemical reaction are treated explicitly according to:

$$
\begin{gathered}
\frac{\partial \phi}{\partial t}=D \frac{\partial^{2} \phi}{\partial x^{2}} \\
\frac{\partial \phi}{\partial t}=\dot{\omega}
\end{gathered}
$$


where $\phi$ is the scalar mass fraction, $\mathrm{D}$ is the diffusion coefficient, and $\omega_{\mathrm{i}}$ is the reaction rate. Since the flow field is resolved in the one-dimensional domain, no modeling is required for molecular mixing and chemical reaction.

The influence of turbulent convection is modeled stochastically by random rearrangements of the scalar field along the domain. Each event involves spatial redistribution of the species field within a specified segment of the linear domain. The rearrangement events are specified by two parameters: $\lambda$, a frequency determining the rate of rearrangement events (stirring), and $\mathrm{f}(1)$, a pdf describing the size distribution of the segments to be rearranged. For high Reynolds number turbulence described by a Kolmogorov cascade, these parameters can be obtained from (McMurtry et al., 1992):

$$
\begin{aligned}
& f(1)=\frac{5}{3} \frac{1^{-8 / 3}}{\eta^{-5 / 3}-L^{-5 / 3}} \\
& \lambda=\frac{54}{5} \frac{v \operatorname{Re}_{\mathrm{L}}}{\mathrm{L}^{3}}\left(\frac{\mathrm{L}}{\eta}\right)^{5 / 3}
\end{aligned}
$$

where $\mathrm{Re}_{\mathrm{L}}$ is the Reynolds number based on the integral length scale, $\mathrm{v}$ is the kinematic viscosity, $\eta$ is the Kolmogorov scale, and $\mathrm{L}$ is the integral scale.

The LEM subgrid mixing and reaction processes are coupled to the large-scale transport in CFDACE+ through the LES resolved flux at each grid cell face through splicing events. Portions of the linear eddy domain are transferred to neighboring grid cells, as shown in Figure 5. These splicing events occur at a frequency determined by the large eddy time step and is much greater than the time step governing the convection-diffusion-reaction process in the subgrid.

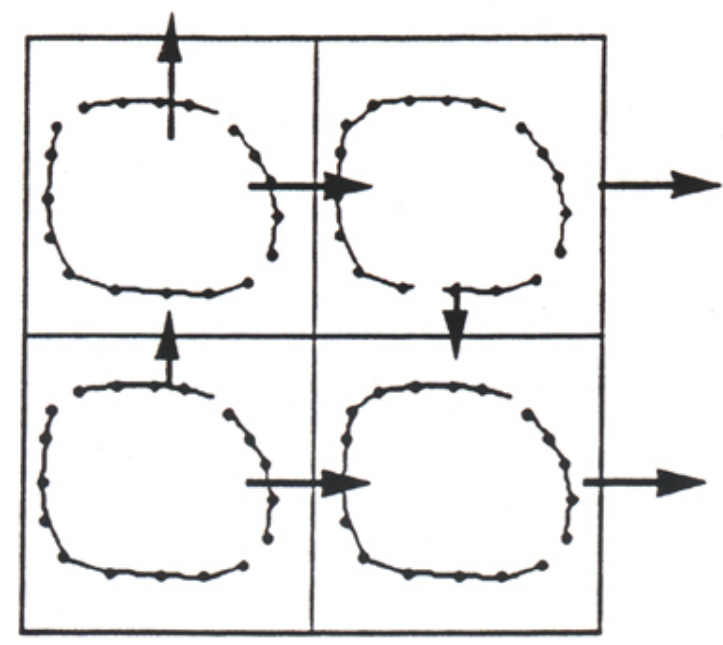

Figure 5. Schematic Diagram of the Linear Eddy Splicing Events 


\subsubsection{LEM Validation}

The LEM was validated against experimental data from the reacting back-step case of Pitz and Daily (1983). The geometry, conditions, and results for the cold flow case were presented in previous reports. The reacting flow case utilized a premixed propane-air flame with an equivalence ratio of 0.57. Figure 6 shows the computational domain and predicted cold flow axial velocity contours. The mean velocity, pressure, and temperature at the inlet are $\mathrm{u} 0=13.3$ $\mathrm{m} / \mathrm{s}, \mathrm{p} 0=1 \mathrm{~atm}$, and $\mathrm{T} 0=293 \mathrm{~K}$ respectively, resulting in a bulk Reynolds number of 22,100. Previously reported cold flow predictions showed good agreement with experimental data. The predicted reattachment length was $6.91 \mathrm{H}$, close to the measured value of $7.0 \mathrm{H}$.

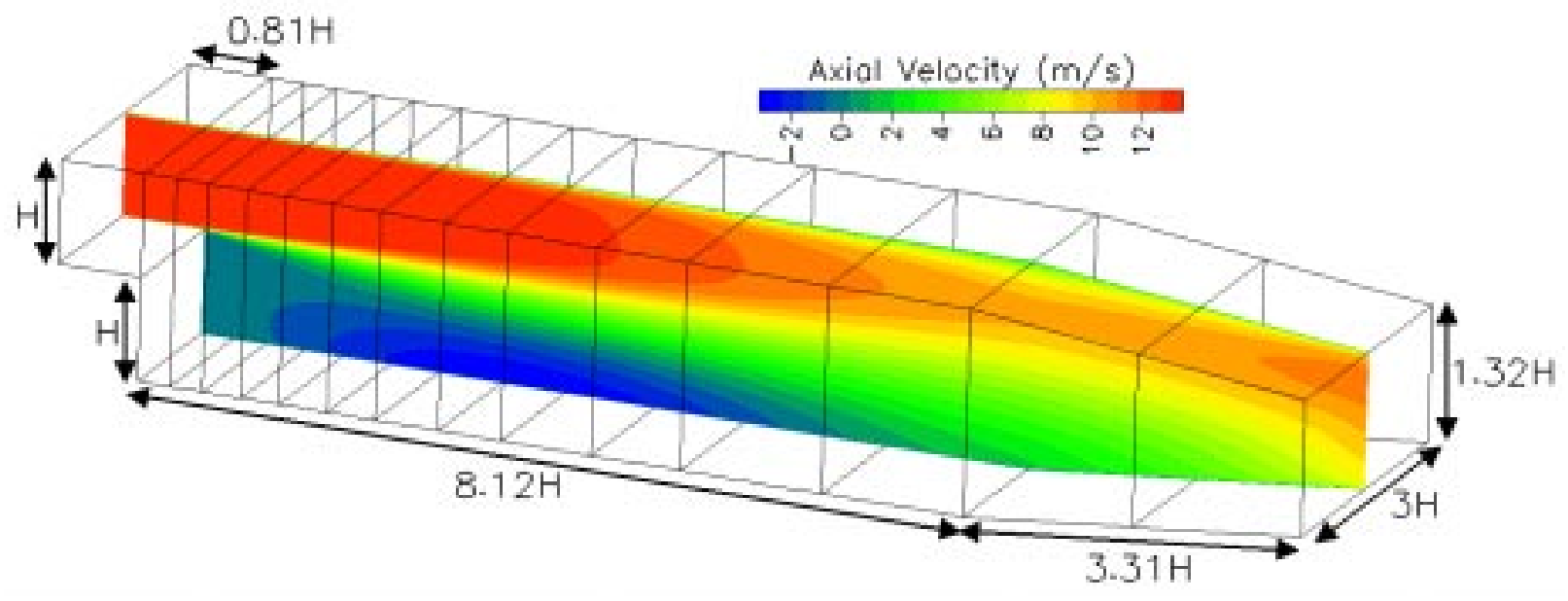

Figure 6. Computational Domain and Non-reacting Axial Velocity Contours for Backstep Case $(H=.0254 \mathrm{~m})$

Reacting flow simulations were carried out using a 1-step propane-air reaction with LEM. The LDKM subgrid turbulence model was used to close the momentum equations at the LES grid level and the subgrid tke provided necessary input for the LEM. For computational efficiency, these initial predictions utilized a 2D planar geometry. A total of 10,132 cells were used with clustering in the shear layer. Wall temperatures were specified according to the experimental data. A converged steady-state solution was utilized as the initial condition for the LES calculations.

The predicted instantaneous flame shape strongly depends on the subgrid chemistry model assumptions. Figure 7 shows instantaneous schlieren images for LES predictions using laminar chemistry and LEM and the measured Schlieren image. The laminar chemistry assumption did not account for subgrid stirring events and was not able to predict large-scale mixing. The LEM results showed significantly more large-scale mixing, in reasonable agreement with the experimental data. The very fine-scale structures do not show up in the predictions since their effects were described with the subgrid turbulence model. 


\section{Laminar Chemistry}

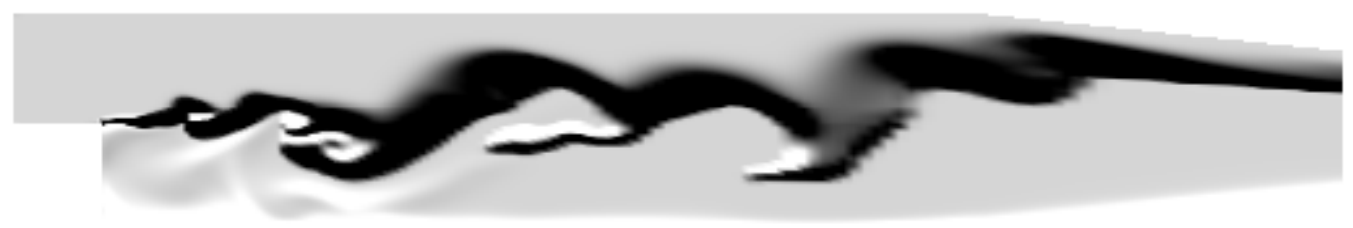

LEM Subgrid Chemistry

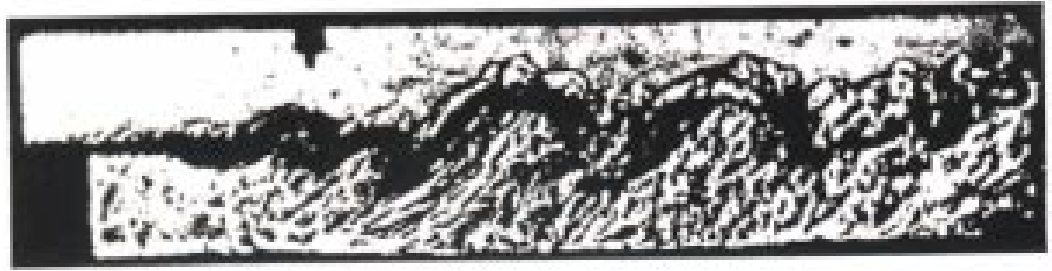

Measured Schlieren Image

Figure 7. Schlieren Images for LES Predictions and Measurements

In this reacting case, the incoming fluid contains cold premixed reactants which mix with hot combustion products in the initial shear layer behind the step prior to burning. The high turbulent strain rates (modeled in the subgrid) should delay the heat release, allowing the development of a Kelvin-Helmholtz instability. With laminar chemistry, the flame speed is independent of strain rate, and strong chemical reaction is allowed to take place immediately after the cold premixture contacts the hot products. The heat release in the shear layer inhibits the growth of the instability, resulting in a near smooth flame surface with very little wrinkling. The LEM includes subgrid turbulent stirring effects and allows the development of a KelvinHelmholtz instability. Figure 8 shows the instantaneous chemical reaction rates for the laminar chemistry and LEM predictions. Not only is the reaction zone delayed downstream of the dump plane for the LEM, but also the reaction flame zone is much wider at certain points and broken in certain points due to subgrid turbulent stirring. 


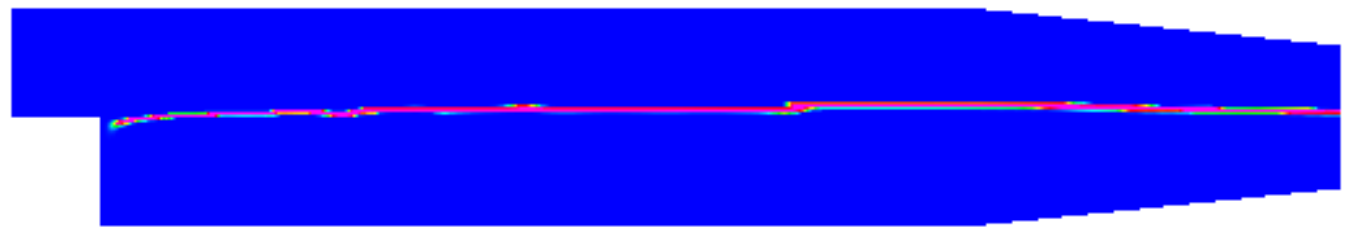

Laminar Chemistry

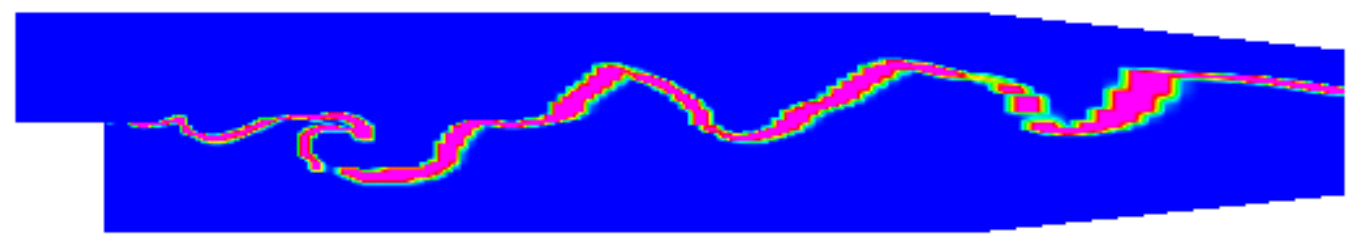

LEM Subgrid Chemistry

Figure 8. Predicted Chemical Reaction Rates

The reattachment length for the reacting flow is much less than the non-reacting case. Figure 9 shows the predicted reattachment length for the laminar chemistry and LEM predictions. The LEM case predicts a value of $3.6 \mathrm{H}$, near the experimental value of $4.5 \mathrm{H}$. The laminar chemistry prediction shows a reattachment length of almost $7.4 \mathrm{H}$. The development of the shear layer vortices are required so the large-scale structures can close down the recirculation zone. These results show the need for accurate subgrid chemistry modeling, particularly in regions where strain rate extinction can occur. It is likely that richer premixed reactants would provide a more stable situation and the laminar chemistry approximation may be more appropriate.

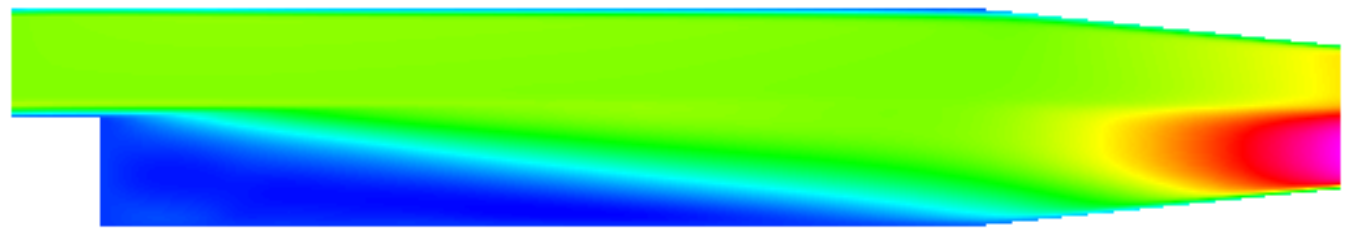

Laminar Chemistry

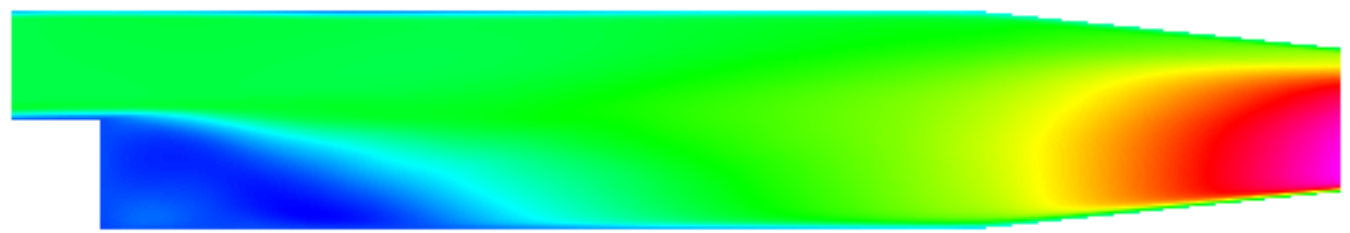

LEM Subgrid Chemistry

Figure 9. Predicted Axial Velocity Contours Showing Reattachment Lengths using Laminar Chemistry and Linear Eddy Model

The computational time for the LEM subgrid and laminar chemistry model were similar. One reason for the relatively fast LEM is due to computing subgrid chemistry once per timestep, instead of at each iteration (7/timestep) for the laminar case. These calculations were performed with a fixed LEM cell number (32) in each LES cell. The effect of this LEM cell number needs to be investigated. Overall these results are very promising, but further work with multi-step chemistry needs to be performed. 


\subsection{Off-Line Linear Eddy Model Using Neural Nets}

Georgia Tech is developing an artificial neural net approach for efficient LEM calculations. For benchmarking the neural net, a baseline calculation of the DOE-HAT combustor is being performed. Next quarter, they plan to use the LEM model in their 3D LES code. More detail of the Georgia Tech work is included in Appendix C.

\subsection{Premixed SimVal Combustor Case}

DOE NETL has established a new experimental combustor design, called SimVal, that will be tested in coming months. The SimVal combustor will provide experimental data that can be used to validate combustion CFD codes, with particular emphasis on understanding combustion instability and variable fuel effects at actual gas turbine combustor conditions. Detailed experimental data will be obtained, including emission images, velocity, temperature, and species maps, and dynamic wall pressure. The current geometry includes a choke plate immediately upstream of the swirl vanes and a choked nozzle at the downstream end of a resonant section. Figure 10 shows the current baseline geometry.

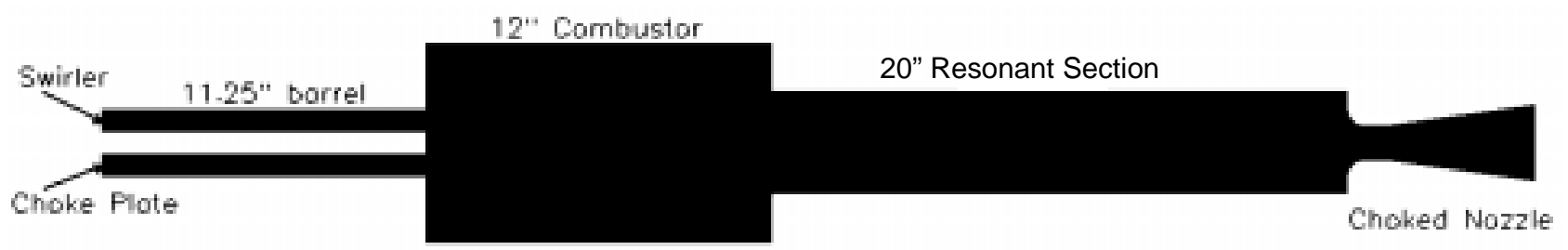

Figure 10. SimVal 2D Axisymmetric Geometry for Baseline Case

Initial transient 2D axisymmetric calculations, including unsteady RANS and LES, were performed at various conditions. These initial simulations provide insight towards instability mechanisms that are driven for the baseline geometry. They also provide further insight on appropriate boundary conditions for LES. Several cases were run with variations in the barrel length, combustor length, equivalence ratio, and turbulence model.

\section{Baseline Case}

The baseline case was simulated at the following conditions:

$$
\begin{aligned}
& \mathrm{PHI}=0.6 \\
& \mathrm{~V}_{\text {noz }}=45 \mathrm{~m} / \mathrm{s} \\
& \mathrm{Swirl} \text { Angle }=45^{\circ} \\
& \mathrm{P}=5.1 \mathrm{~atm} \\
& \mathrm{~T}_{\text {in }}=533 \mathrm{~K} \\
& \mathrm{~T}_{\text {wall }}=700 \mathrm{~K}
\end{aligned}
$$

A fixed mass flow with completely premixed reactants was assumed at the inlet with a constant swirl angle. The fully burned conditions provided a choked flow at the nozzle throat. An extrapolated boundary condition was used at the supersonic nozzle exit. A total of 12,000 cells was used for unsteady RANS and 65,000 cells for LES. Timesteps of 1E-5 and 3E-6 seconds 
were used for unsteady RANS and LES respectively. The calculations were performed on the parallel Linux PC cluster using between 6 and 8 processors.

Steady-state predictions for the baseline case are shown in Figure 11. The steady results show the expected swirl stabilized flame with central and outer recirculation zones. The swirling flow attaches to the outer combustor wall before flowing out through the resonant section and into the choked nozzle. Transient predictions were initially performed with unsteady RANS, starting from the steady-state initial conditions. Figure 12 shows the predicted pressure history monitored at the combustor dome face. A dominant frequency of $400 \mathrm{~Hz}$ was predicted at an amplitude of $\sim 1.9 \%$ of the mean pressure. Harmonics of that frequency were observed at very low amplitudes. The fundamental longitudinal frequency for the hot gas combustor/resonant section is $\sim 510 \mathrm{~Hz}$. The quarter wave frequency for the cold gas barrel is $\sim 406 \mathrm{~Hz}$. A likely driver of instability in this system would be the coupling of these two modes that provides a strong variation in mass flow rate entering the combustor and flamezone. The predicted frequency of the system corresponds to the natural quarter wave frequency of the cold gas barrel. A series of runs were then performed with different lengths of the barrel.

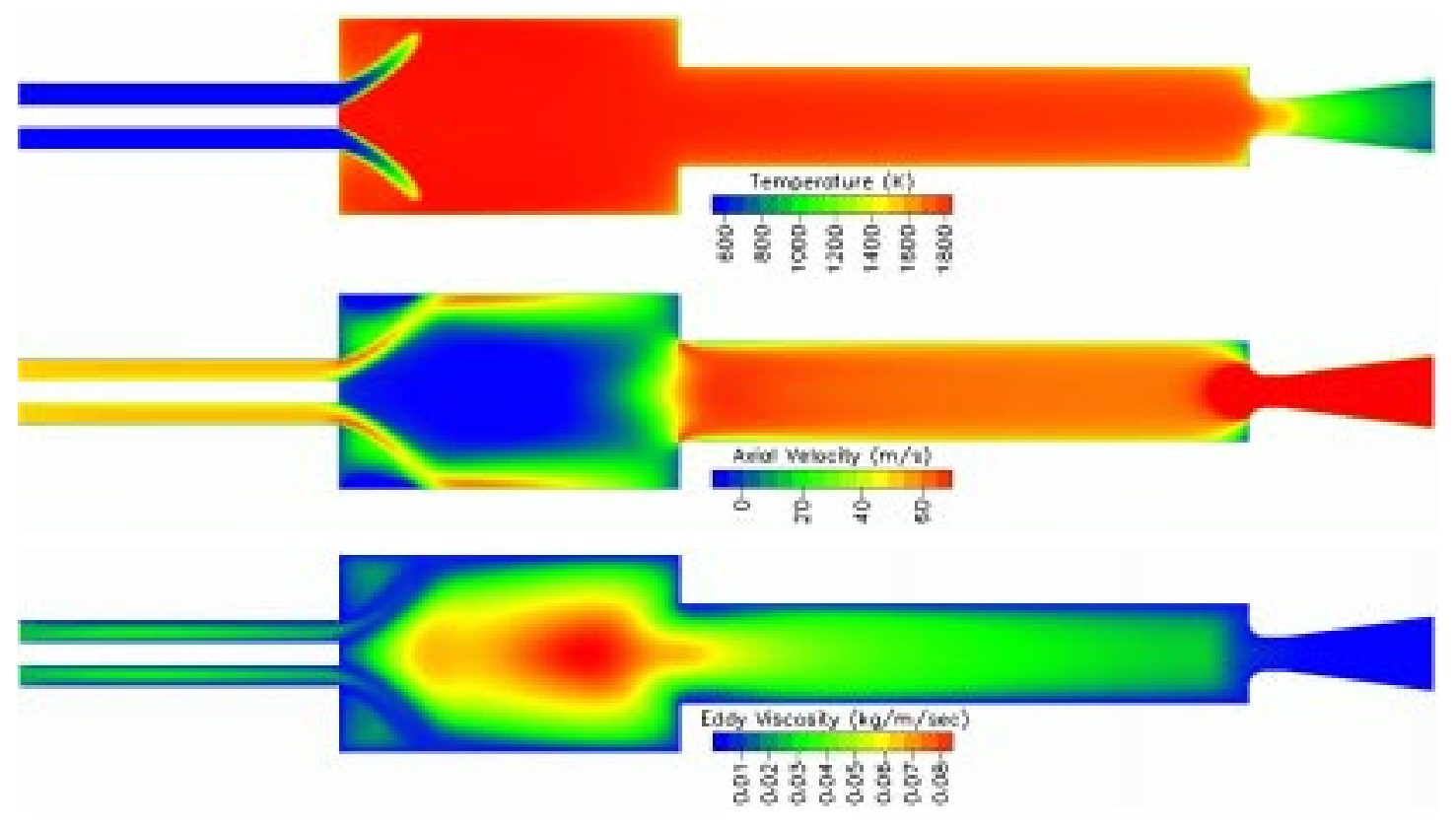

Figure 11. Steady-state Predictions of Temperature, Axial Velocity, and Eddy Viscosity for Baseline SimVal Case 

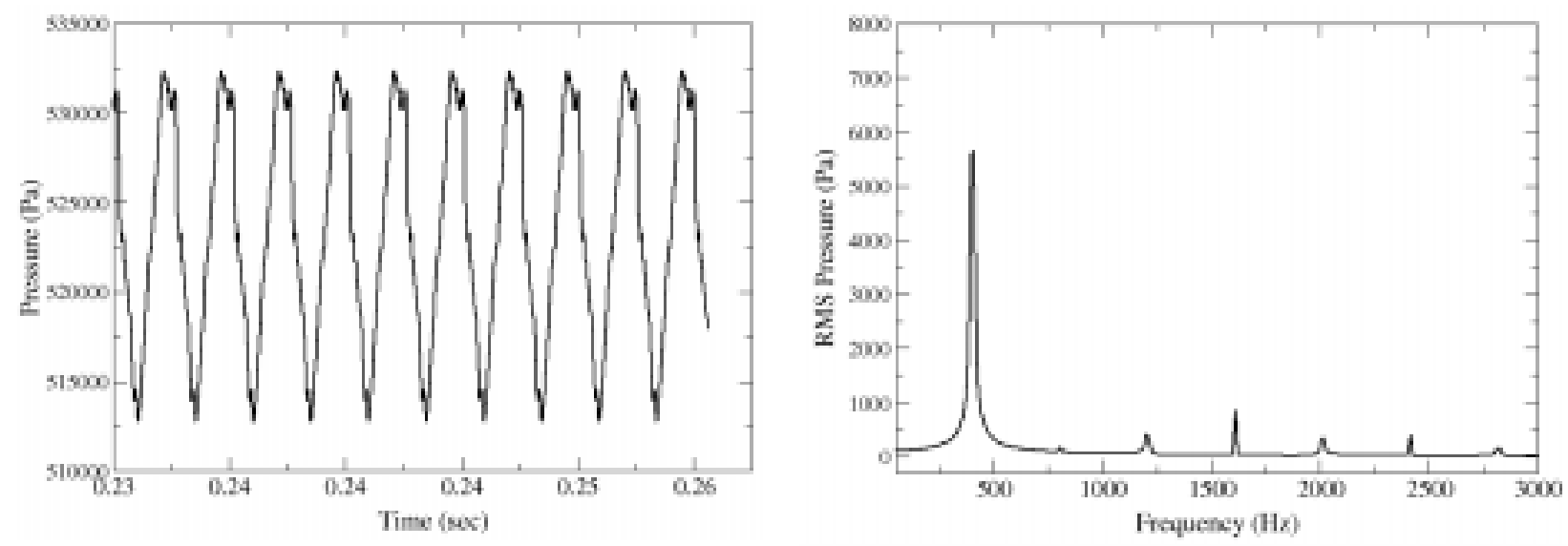

Figure 12. Time History and Fourier Transformed Signal of Predicted Combustor Pressure at Dome Face for Baseline SimVal Case

Unsteady RANS simulations were performed for cases with barrel lengths between 4.75" and 16". Table 2 shows the predicted oscillations from the unsteady RANS calculations with the different barrel lengths. The amplitudes of oscillation observed in these cases are less than has been observed in the past for instabilities driven by equivalence ratio modulation (Richards and Janus, 1997). The largest amplitude oscillation was observed in the baseline case. The smallest amplitude oscillation was observed in the 6.75" barrel case. The 6.75" case also showed two strong frequencies, 1023 and $512 \mathrm{~Hz}$. The higher 1023 frequency exhibited higher peak-to-peak amplitudes. These results indicate that oscillations tend to occur near the fundamental frequency of the combustor/resonant section and its $2^{\text {nd }}$ harmonic. Figure 13 shows the time series and fourier transform of the combustor pressure for Case 2. The occurrence of strong harmonics is observed. The oscillation frequency increases as the barrel length is shortened. A significant jump to the $2^{\text {nd }}$ harmonic $(\sim 1020 \mathrm{~Hz})$ occurs at the two shortest barrel lengths.

Table 2. Predicted Oscillations for Varying Barrel Lengths

\begin{tabular}{|l|c|c|l|}
\hline & Barrel Length & Oscillation Amplitude & \multicolumn{1}{c|}{ Frequency } \\
\hline Case 1 & $16.0 "$ & $0.8 \%$ & $405 \mathrm{~Hz}$ (weak harmonics) \\
\hline Baseline & $11.25^{\prime \prime}$ & $1.9 \%$ & $401 \mathrm{~Hz}$ (weak harmonics) \\
\hline Case 2 & $8.75^{\prime \prime}$ & $1.2 \%$ & $457 \mathrm{~Hz}$ (strong harmonics) \\
\hline Case 3 & $6.75^{\prime \prime}$ & $0.6 \%, 0.3 \%$ & $1023 \mathrm{~Hz}, 512 \mathrm{~Hz}$ (strong harmonics) \\
\hline Case 4 & $4.75^{\prime \prime}$ & $1.1 \%$ & $1027 \mathrm{~Hz}$ (weak harmonics) \\
\hline
\end{tabular}



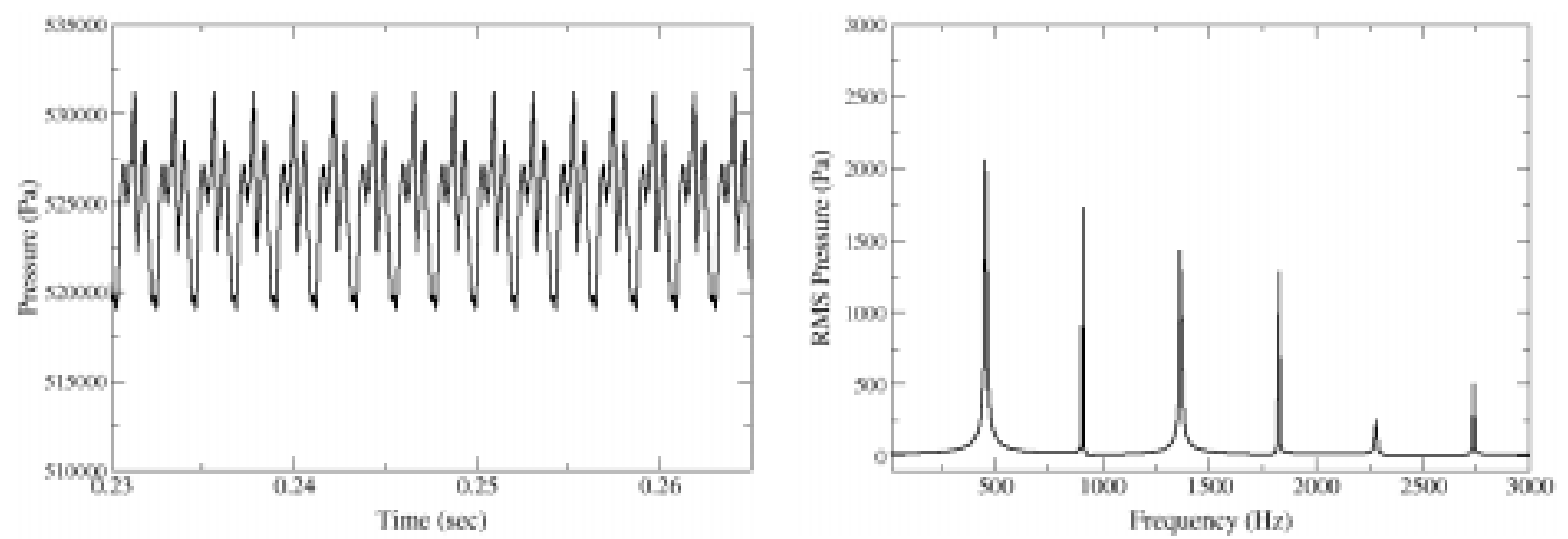

Figure 13. Predicted Time Series and Fourier Transform of Combustor Pressure for Case 2

In addition to trying different barrel lengths, the effect of the resonant section was studied. A case without a combustor neck was tested. This new geometry is shown in Figure 14, where the neckdown is replaced with an extended combustor length. This new straight-pipe combustor case utilized a 16" barrel neck. Figure 15 shows the predicted time series and fourier transform of the combustor pressure. A very high oscillation with an amplitude of $11.5 \%$ of the mean pressure and a frequency of $464 \mathrm{~Hz}$ was predicted. Higher order harmonics were also observed. The frequency of this oscillation corresponds closely to the longitudinal mode of the combustor. The presence of the original resonant section damps the instability significantly. The original 12" combustor has a longitudinal mode frequency of $\sim 1300 \mathrm{~Hz}$. It is possible that this higher frequency mode helps cancel out some of the lower frequency oscillation, making the system more stable. The original resonant section also provides a small pressure drop in the system as the flow is accelerated. This small pressure drop and the smaller effective combustor volume (or mass) may help avoid the larger pressure oscillations.

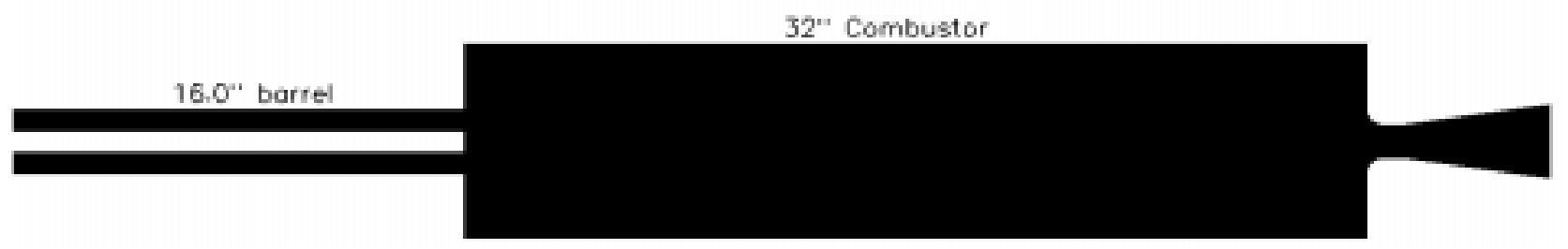

Figure 14. Geometry for Long Pipe Combustor Case 

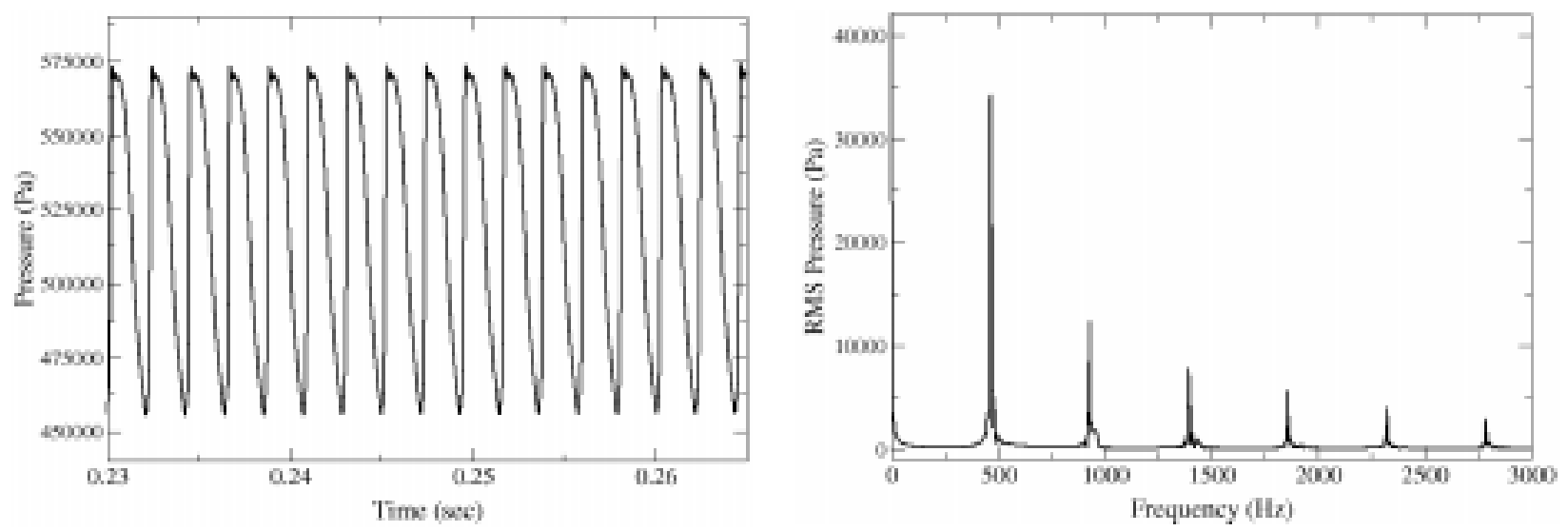

Figure 15. Predicted Time Series and Fourier Transform of Combustor Pressure for Combustor Pipe Case

It is interesting to observe the different flameshapes that are predicted during the oscillation for the large-amplitude instability and the smaller instability. Figures 16 through 19 show predicted instantaneous temperature, axial velocity, heat release, and pressure contours during the cycle (min and max pressure) for the combustor pipe case and Case 4. Significantly more motion occurs during the straight pipe instability. Despite the high amplitude oscillations in the straight pipe case, the flow does not go up inside the barrel at any time during the instability. Previous calculations of the old DOE-NETL case with unchoked barrel flow and equivalence ratio modulation showed significant backflow into the barrel ID during the high pressure portion of the cycle. From the pressure results, it appears that the resonant section adds one or more additional higher frequency modes, so that a single $\sim 500 \mathrm{~Hz}$ mode is not as easily excited. The flow changes induced from the resonant section definitely plays a strong role in the potential for developing combustion instability. It seems likely that the barrel length would not have a large impact on the instability for the straight-pipe combustor. These results indicate that the prudent use of neckdown sections creating regions of acceleration and different acoustic modes in the combustor is an effective way of eliminating longitudinal-mode combustion instabilities. One needs to be careful that an effective Helmholtz resonator is not then created for allowing bulkmode instabilities. The theoretical Helmholtz resonator frequency for Case 4 is $\sim 173 \mathrm{~Hz}$ and was not excited at the simulated conditions. 
$\operatorname{Max}$

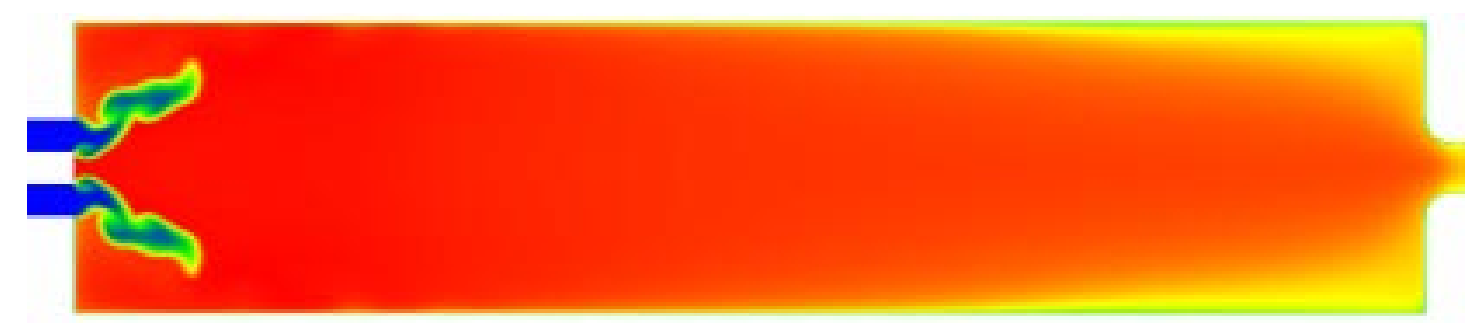

Pressure

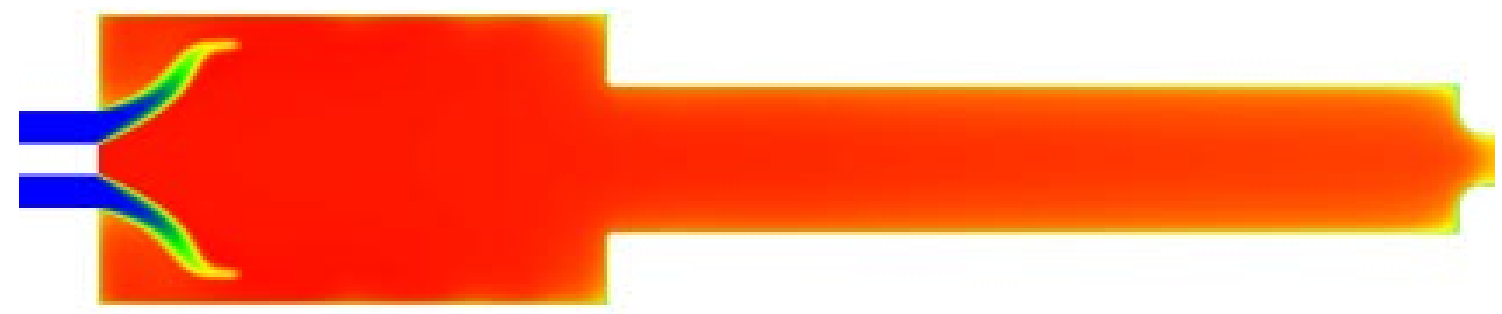

Min

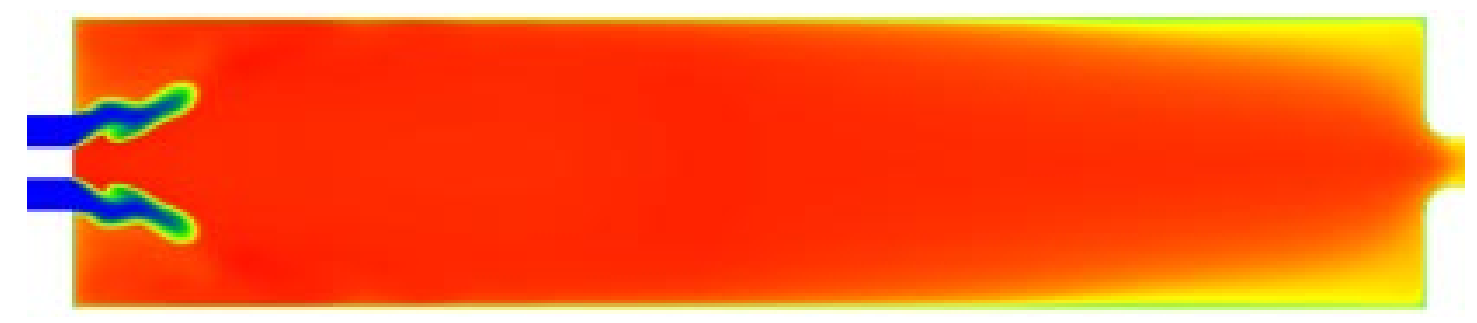

Pressure
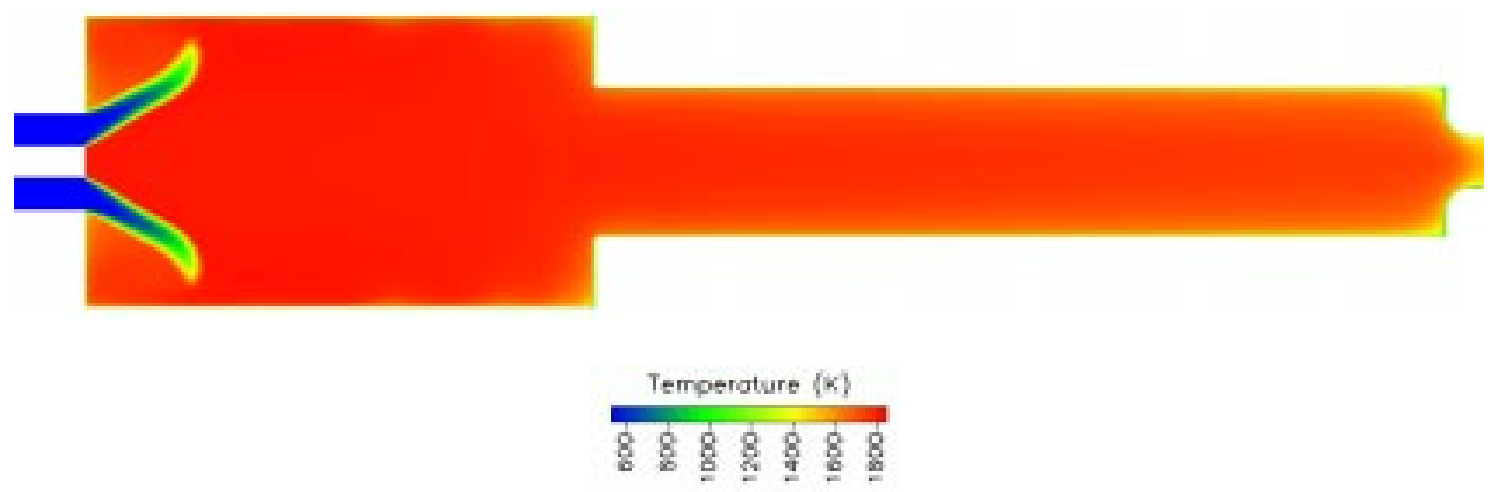

Figure 16. Predicted Instantaneous Temperature Contours at Max and Min Pressure During Instability Cycle for Straight-pipe and Resonant Section Combustor Cases 
Max

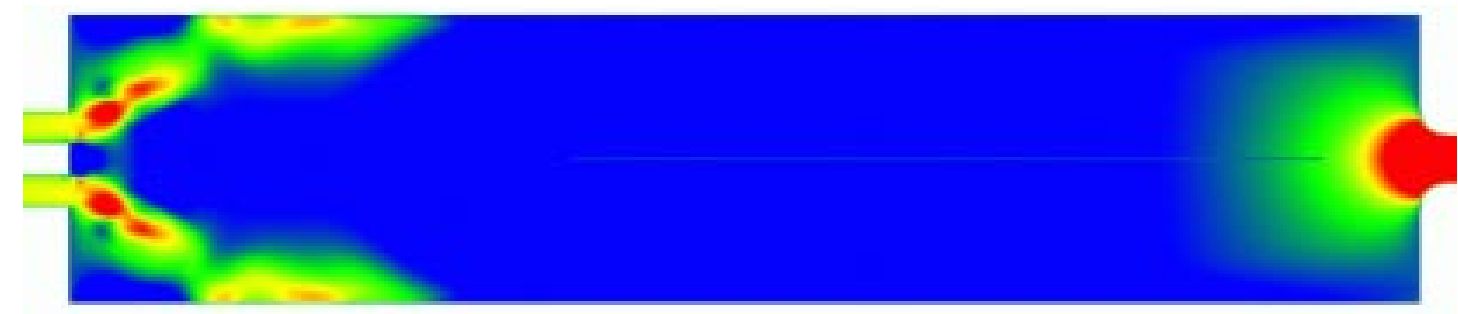

Pressure

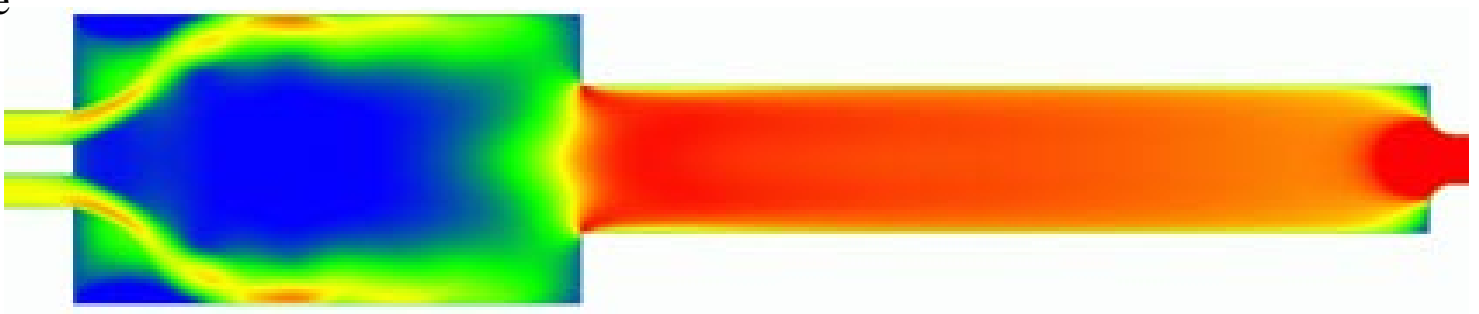

Min

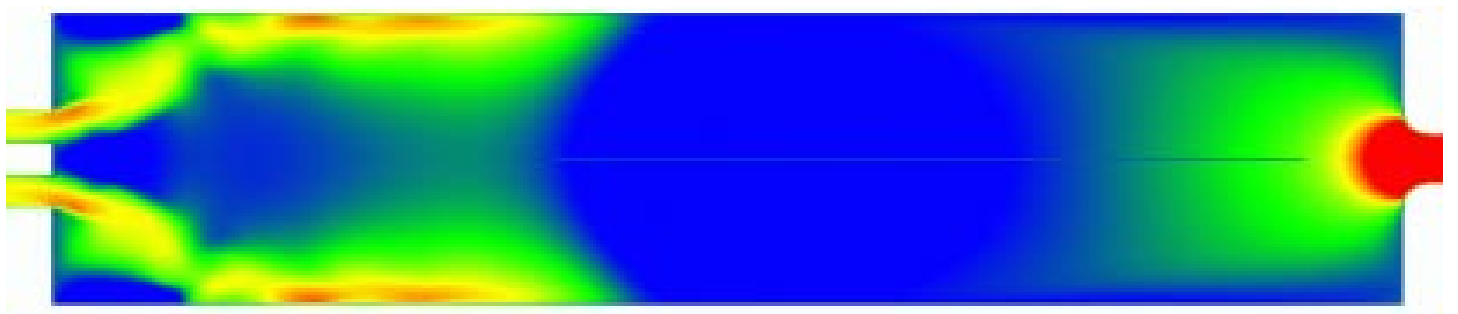

Pressure
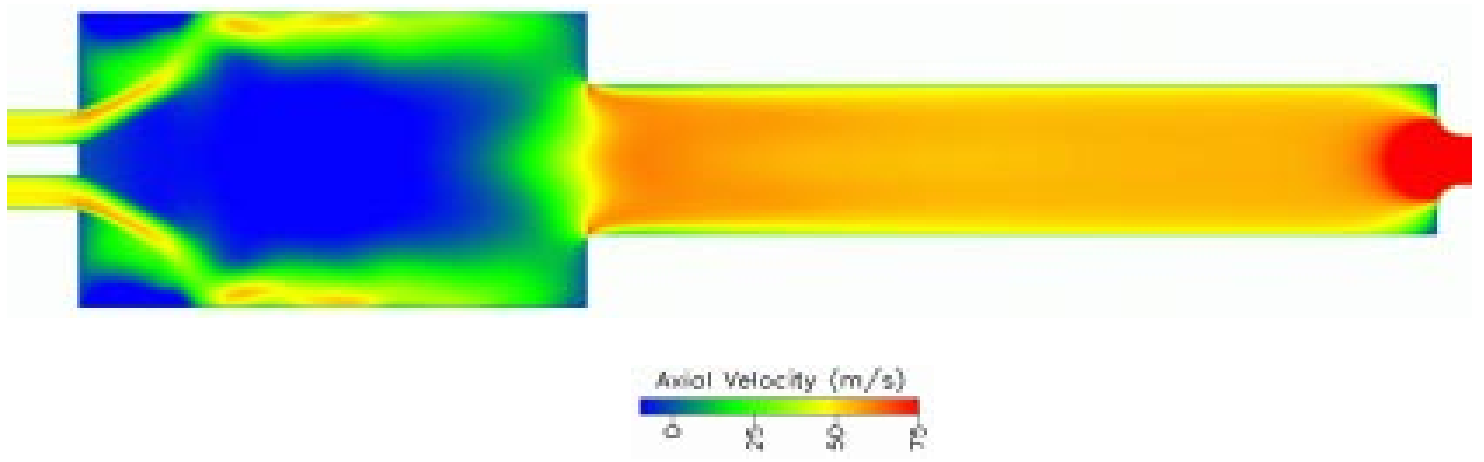

Figure 17. Predicted Instantaneous Axial Velocity Contours at Max and Min Pressure During Instability Cycle for Straight-pipe and Resonant Section Combustor Cases 
Max

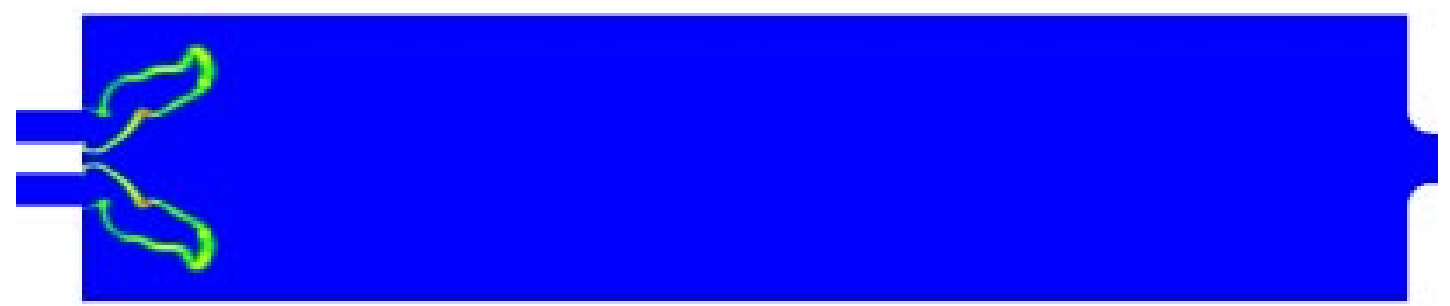

Pressure
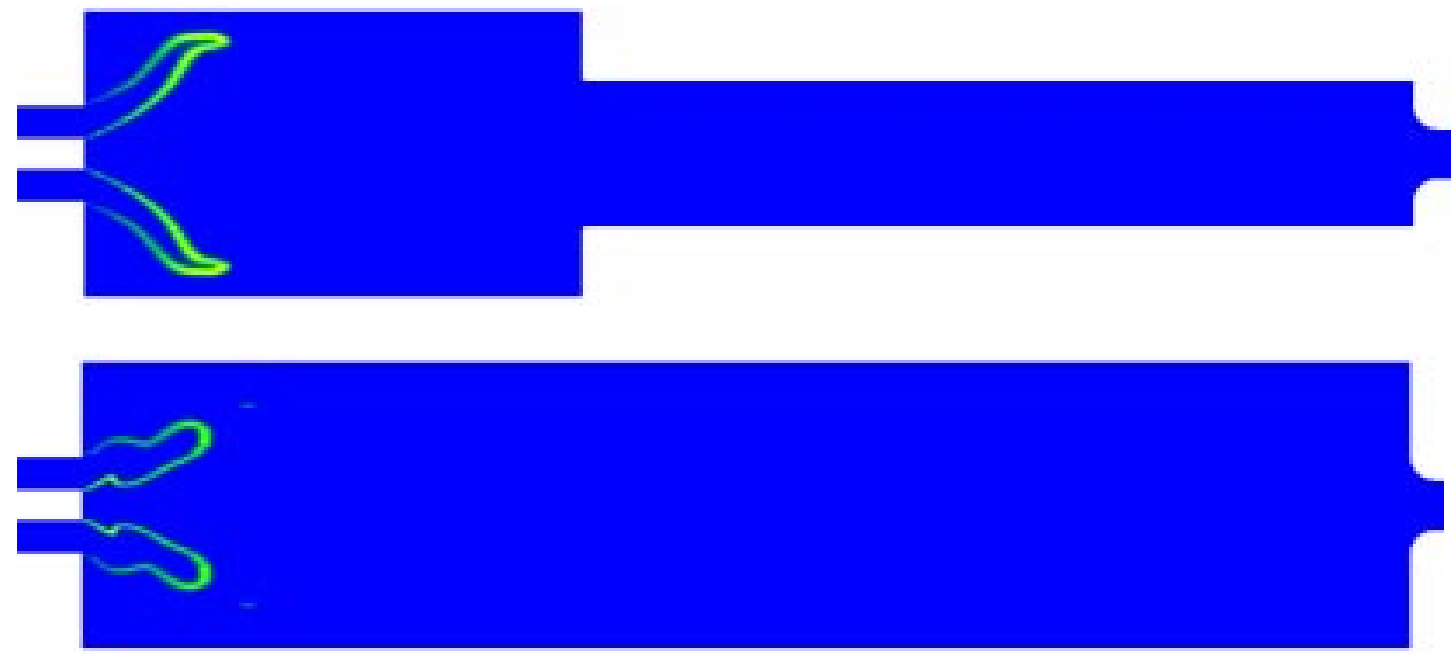

Min

Pressure

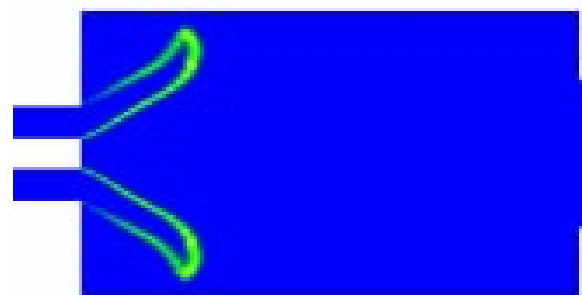

Heot fieleose

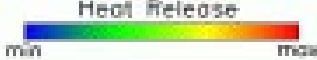

Figure 18. Predicted Instantaneous Heat Release Contours at Max and Min Pressure During Instability Cycle for Straight-pipe and Resonant Section Combustor Cases 

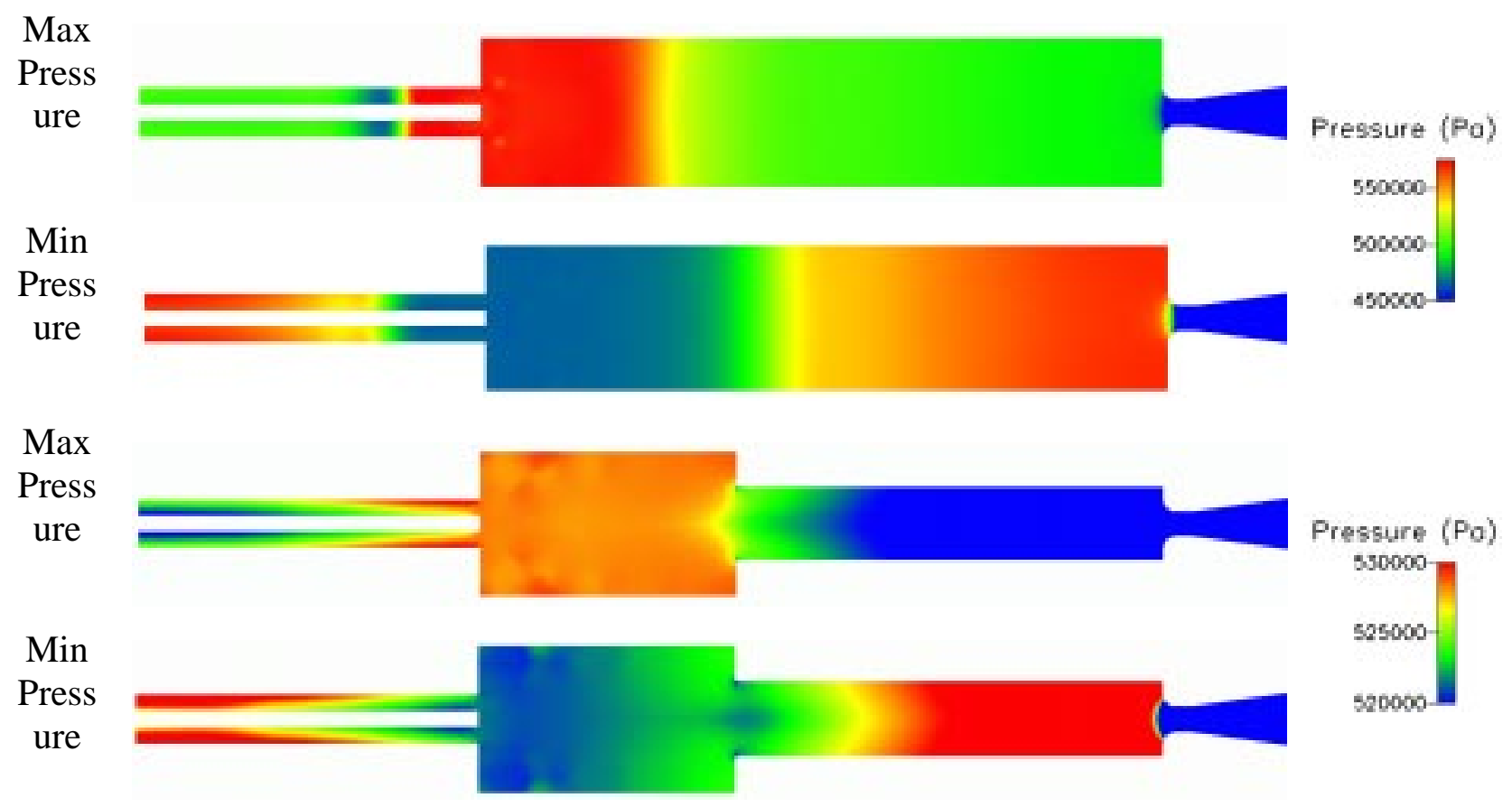

Figure 19. Predicted Instantaneous Pressure Contours at Max and Min Pressure During Instability Cycle for Straight-pipe and Resonant Section Combustor Cases

Several cases were also simulated at different fuel-air equivalence ratios. Table 3 presents the oscillation results for cases with different fuel-air equivalence ratios. All equivalence ratio cases were run with the baseline geometry. The low amplitude oscillations for the leanest case seems to be from spreading the flame out significantly in the axial direction. A spatially distributed heat release zone should always help minimize acoustic/heat release coupling.

Table 3. Predicted Oscillations for Varying Mixture Equivalence Ratio

\begin{tabular}{|l|c|c|l|}
\hline & Equivalence Ratio & Oscillation Amplitude & \multicolumn{1}{c|}{ Frequency } \\
\hline Case 5 & 0.5 & $0.2 \%$ & $440 \mathrm{~Hz}$ (weak harmonics) \\
\hline Baseline & 0.6 & $1.9 \%$ & $401 \mathrm{~Hz}$ (weak harmonics) \\
\hline Case 6 & 0.7 & $1.1 \%$ & $447 \mathrm{~Hz}$ (strong 3 ${ }^{\text {rd }}$ harmonic) \\
\hline Case 7 & 0.8 & $0.9 \%$ & $468 \mathrm{~Hz}$ (strong 3 ${ }^{\text {rd }}$ harmonic) \\
\hline
\end{tabular}

Final calculations were performed by utilizing LES models for describing the subgrid turbulence. In order to achieve adequate turbulence levels inside the barrel, high speed jets were introduced at the swirler inlet boundary. Without the turbulent jets in LES mode, significant recirculation along the barrel ID (up to the swirler) occurred. Table 4 shows the results using unsteady RANS and LES with the Smagorinsky and LDKM subgrid turbulence models. These results are 
interesting since they show not much effect of turbulence model on the lower frequency oscillation. The important test will be to include chemistry-turbulence interactions and capture the effect of subgrid stirring on the reaction. Results with the LEM should be finished for next quarter.

Table 4. Predicted Oscillations for Unsteady RANS and LES

\begin{tabular}{|l|c|c|l|}
\hline & Turbulence Model & Oscillation Amplitude & \multicolumn{1}{|c|}{ Frequency } \\
\hline Case 8 & Unsteady RANS & $1.8 \%$ & $397 \mathrm{~Hz}$ (weak harmonics) \\
\hline Case 9 & Smagorinsky & $1.8 \%$ & $411 \mathrm{~Hz} \& 1359 \mathrm{~Hz}$ \\
\hline Case 10 & LDKM & $2.3 \%$ & $411 \mathrm{~Hz} \& 1341 \mathrm{~Hz}$ \\
\hline
\end{tabular}

Despite the similarities in capturing the low frequency oscillation, the LES cases predicted higher frequency oscillations compared to the unsteady RANS. Figure 20 shows the predicted temperature contours at max and min pressure for the unsteady RANS and LES with LDKM. The LES case predicts smaller structures and pockets of cold reactants that tear away from the main unburned fluid. Also, the LES case allows hot products to recirculate back along the barrel ID during part of the cycle. These calculations need to be performed with more detailed chemistry to predict emissions and with subgrid chemistry models to capture subgrid turbulencechemistry interactions.
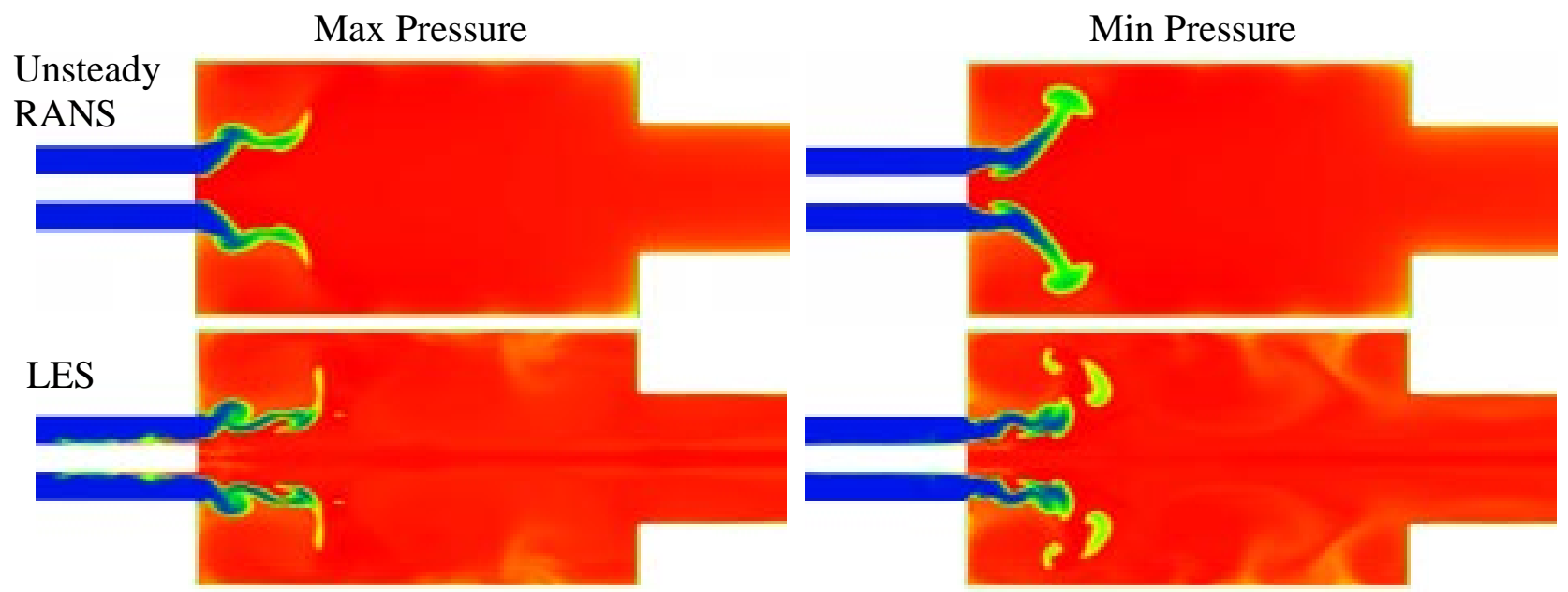

Figure 20. Predicted Instantaneous Temperature Contours at Max and Min Pressure for the Unsteady RANS and LES with LDKM 


\section{CONCLUSION}

The combustion LES code has been further developed and tested for predicting turbulent reacting flows. The LES code can now use the LEM to better represent turbulent reacting flow since subgrid stirring effects are now resolved. The ISAT has been refined and optimized and gives a 2-3 speedup using the 9 species mechanism derived from the full GRI. The artificial neural net (ANN) approach has been demonstrated in Georgia Tech's single cell LES code but needs to be implemented and tested in CFD-ACE+. The LES code has undergone initial testing with the premixed SimVal baseline combustor.

\section{REFERENCES}

Maloney, D., (2001), Personal Communication, DOE-NETL.

McMurtry, P.A., Menon, S. Kerstein, A.R., (1992), "A Linear Eddy Sub-grid Model for Turbulent Reacting Flows: Application to Hydrogen-Air Combustion," Twenty-Fourth Symposium (International) on Combustion/The Combustion Institute, pp. 271-278.

Pitz, R.W. and Daily, J.W., (1983), "Combustion in a Turbulent Mixing Layer Formed at a Rearward-Facing Step," AIAA J. 21 pp.1565 --1570.

Pope, S.B., (1997), "Computationally Efficient Implementation of Combustion Chemistry Using In Situ Adaptive Tabulation," Combustion Theory and Modeling, Vol. 1, pp. 41-63.

Richards, G.A. and Janus, M.C., (1997), "Characterization of Oscillations During Premix Gas Turbine Combustion," Presented at the International Gas Turbine and Aeroengine Congress and Exhibition, 97-GT-244, Orlando, FL, June 2-5, 1997. 


\section{APPENDIX A - WORK SCHEDULE}

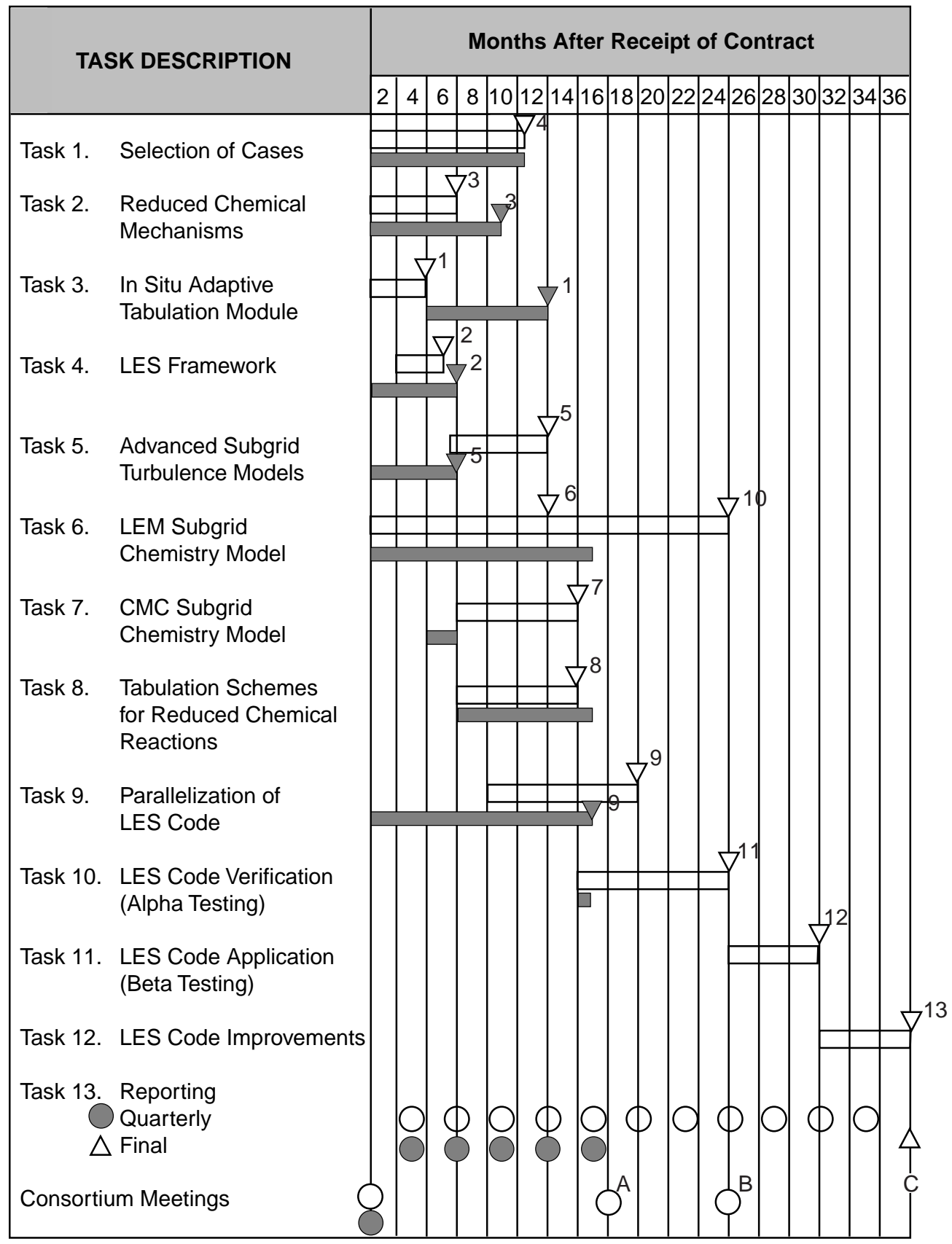

Key Milestones

1 Complete In-Situ Adaptive Tabulation Module

2 Complete LES Framework Modification to CFD-ACE+

3 Complete Reduced Mechanisms

4 Complete Selection of Cases

5 Complete Implementation of Turbulence Models

6 Complete Implementation of Initial Version of LEM Model

7 Complete Implementation of CMC Model

8 Complete Tabulation Schemes

9 Complete Parallelization of LES Code 10 Complete Implementation of LEM Model 11 Complete Alpha Testing of LES Code 12 Complete Beta Testing of LES Code 13 Final Release of LES Code

\section{Performance Targets}

A Alpha Release of LES Code

B Beta Release of LES Code

C Final Commercial Release of LES Code 


\section{APPENDIX B - FUTURE PLANS}

During the next quarter, the following work is planned:

1. Optimize and refine ISAT for large chemical mechanisms (> 19 species).

2. Carry out initial validation of LES code for predicting emissions and instability in selecting cases from industrial consortium.

3. Develop, implement, and test neural net on lean premixed SimVal combustor test case. 
APPENDIX C - GEORGIA TECH PROGRESS 


\title{
LES of Premixed Combustion in the DOE-HAT Combustor
}

\author{
S. Menon and G. Eggenspieler \\ School of Aerospace Engineering \\ Georgia Institute of Technology \\ ATLANTA, GA
}

January 27, 2002

Quarterly Report For Project E-16-T04

Submitted to

CFD Research Corporation

\section{Introduction}

Recent emission regulations have pushed for the development of more fuel efficient and low- $\mathrm{NO}_{x}$ gas turbine systems. Thus, design studies of new device will require accurate prediction of emission as a function of the operating conditions. For example, recent measurements in a full-scale combustor (denoted DOE-HAT hereafter) [Bhargava et al., 2000] showed that as the equivalence ratio is decreased the CO emission first decreases and then, suddenly increases. RANS codes have not been successful in capturing this trend. Therefore, a LES model is being developed with the goal of predicting the emission from typical gas turbine combustor. The eventual goal of this project is to develop a computationally efficient approach to predict emissions from such combustor. In order to do this, we will develop and implement an Artificial Neural Network based chemistry lookup approach within the LES formulation.

However, before implementing this approach, it is necessary to demonstrate the LES can be used to predict pollutant emission accurately and also that LES can predict the $\mathrm{CO}$ variation with equivalence ratio. To do this, we are implementing the subgrid combustion model developed earlier to study this flow. A two-step mechanism with $\mathrm{CO}$ kinetics is implemented into the code, as described below. We compare this approach with the classical G-equation model where the chemistry is not modeled and the flame is tracked as a thin interface that separates the premixed reactants from the product.

We present the numerical method used for the LES in this study in the next section and this is followed by the G-equation and finite-rate kinetics modeling used 
in this study. In Section 4 we present the results obtained so far and in Section 5 we note the current and future work plan for the next two quarters.

\section{Numerical method}

In the past LES studies of dump combustor, a cylindrical grid was employed which results in a centerline singularity. This centerline problem was avoided by a modification of the flux calculation at the centerline. The key advantage of this grid configuration is that regions of high shear in the separated shear layer can be resolved using body conforming grid. The centerline modification used in the past works reasonably well when there is very little cross flow across the centerline. For the earlier studies of a dump combustor, this assumption was valid but for the DOE-HAT combustor, the central plug causes significant flapping of the shear layer and thus, there is significant normal transport of momentum. In order to capture this behavior, it is necessary to resolve the centerline flow region more accurately and without any special constraints.

\section{$2.1 \quad$ Overlapping Grid for LES}

In order to solve this problem while at the same time maintaining accuracy in the circular shear layer region at the dump plane, we have developed an overlapping grid that combines the cylindrical-type grid with a cartesian-type grid. The communication between these two grids is performed using an interpolation technique. The grid is shown in Fig. 1 and 2. Figure 1 is a global view of the grid mesh and Fig. 2 shows a front view of the grid mesh in the centerline region.

The information required by the cylindrical grid are interpolated using the values from the cartesian grid (and vice versa). This interpolation is nominally second-order accurate in both directions at present provided the grid distribution and stretching is kept within acceptable values. We are also exploring another approach that will increase this interpolation to fourth or fifth order. Such high accuracy will be needed for cases when the LES employs a fourth-order accurate algorithm.

In the current implementation, the interpolated value $\mathrm{q}$ is obtained by using the following formula.

$$
\begin{aligned}
& q_{p}=q_{j, k}+\mathrm{a} \Delta^{1,0} q_{j, k}+\mathrm{b} \Delta^{0,1} q_{j, k} \\
& +\frac{A}{2 !}\left(\mathrm{a}(\mathrm{a}-1) \Delta^{2,0} q_{j, k}+2 \mathrm{ab} \Delta^{1,1} q_{j, k}+\left(\mathrm{b}(\mathrm{b}-1) \Delta^{0,2} q_{j, k}\right)\right.
\end{aligned}
$$

where:

$$
\begin{aligned}
& \mathrm{a}=\frac{x_{p}-x_{j, k}}{h}, \mathrm{~h}=x_{j+1, k}-x_{j, k} \\
& \mathrm{~b}=\frac{y_{p}-y_{j, k}}{f}, \mathrm{f}=y_{j+1, k}-y_{j, k} \\
& \Delta^{1,0} q_{j, k}=q_{j+1, k}-q_{j, k} \\
& \Delta^{0,1} q_{j, k}=q_{j, k+1}-q_{j, k} \\
& \Delta^{2,0} q_{j, k}=q_{j+2, k}-2 q_{j+1, k}+q_{j, k} \\
& \Delta^{0,2} q_{j, k}=q_{j, k+2}-2 q_{j, k+1}+q_{j, k} \\
& \Delta^{1,1} q_{j, k}=q_{j+1, k+1}-q_{j+1, k}-q_{j, k+1}+q_{j, k}
\end{aligned}
$$


The interpolation scheme is second order if $\mathrm{A}=1$, first if $\mathrm{A}=0$.

Since our code is also MPI parallel, in addition to proper interpolation, implementation in parallel has to be optimized to increase computational efficiency. This has been successfully achieved in the last quarter.

For the study reported here, a grid resolution of 130x80x81 is used for the cylindrical grid and 134x20x20 for the cartesian grid. Significant clustering is used in the regions of high shear. The inlet region is also resolved with grid clustering near the wall to resolve the boundary layer.

\subsection{Thin Flame Approach}

To track the flame without using finite-rate kinetics, a flame front tracking model is employed. In this approach, the flame front is tracked as a infinitely thin surface that is convected by the flow and also propagates normal to itself at a characteristic flame speed $S_{f}$. In laminar flow, $S_{f}=S_{L}$, where $S_{L}$ is the laminar flame speed, which contains within it all the thermochemical state of the fuel-air mixture. For a given equivalence ratio, $S_{L}$ is well defined. However, in turbulent flow, the turbulent flame speed $S_{T}$ is still required to be modelled. Typically, $S_{T}=S_{T}\left(u^{\prime}, S_{L}\right)$, where $u^{\prime}$ is subgrid turbulence intensity. In the present study, a model employed in our past studies for gas turbine simulations [Kim et al., 1999, Kim and Menon, 2000] is used to obtain the turbulent flame speed as a function of $u^{\prime}$ (which is obtained using the subgrid kinetic energy, $k^{s g s}$ ) and $S_{L}$.

The mathematical model for the flame front considers a progress variable $G$ which is governed by a transport equation which is of the following form:

$$
\frac{\partial \rho G}{\partial t}+\frac{\partial \rho G u_{i}}{\partial x_{i}}=-\rho_{0} S_{T}|\nabla G|
$$

Here, $\rho_{0}$ is the reference density in the reactant mixture and $\rho$ is the LES filtered density. Also, $G$ and $u_{i}$ are respectively, the LES-resolved progress variable and flow velocity. The last term represents a closure of the convective subgrid flux related to the LES filtering. It is modelled using a gradient diffusion at the resolved scale and $D_{G}$ is the effective subgrid diffusion which is obtained using the subgrid eddy viscosity model (which is also used for the momentum transport closure) and using a turbulent Schmidt number of unity. Further details are in the cited references.

Note that in the present implementation, the LES-resolved variable $G$ can range from 0 to 1 and represents a measure of the flame brush thickness. This is in contrast to the original "laminar" interpretation of the $G$ field where $G=0$ denotes burned product and $G=1$ denotes reactants and the flame is an infinitely thin interface between $G=0$ and $G=1$. In the LES implementation the filtered $G$ represents the resolved flame brush which is an average location of the instantaneous thin flames. The flame brush thickness can be determined by estimating the variance

$G^{2}$ [Peters, 2000]. It should be noted that Eq. (1) is valid only for the corrugated flamelet regime where the flame thickness is smaller than the Kolmogorov scale. 


\subsection{Finite-Rate Kinetics Approach}

The G-equation model allows us to determine the flame location and characteristics without using finite rate chemistry. However, it cannot provide information on pollutant formation, such as CO emission. Although detailed or skeletal mechanism is needed for accurate prediction, implementation of such detailed kinetics into LES would result in computational cost that is currently considered too excessive. It is anticipated that the ANN method being developed will provide a computationally efficient approach to include detailed kinetics into LES. In the meantime, to demonstrate the integration of finite-rate kinetics into LES, we begin with a two-step mechanism for propane as proposed by Westbrook and Dryer and combine this a subgrid eddy breakup model to achieve a LES closure. The mechanism we are currently employing is the following:

$$
\begin{gathered}
\mathrm{C}_{3} \mathrm{H}_{8}+3.5\left(\mathrm{O}_{2}+3.76 \mathrm{~N}_{2}\right) \rightarrow 3 \mathrm{CO}+4 \mathrm{H}_{2} \mathrm{O}+13.16 \mathrm{~N}_{2} \\
\mathrm{CO}+0.5 \mathrm{O}_{2} \rightarrow \mathrm{CO}_{2} \\
\mathrm{CO}+0.5 \mathrm{O}_{2} \leftarrow \mathrm{CO}_{2}
\end{gathered}
$$

where the last two reactions are the $\mathrm{CO}$ consumption and formation mechanisms, respectively. The Arhenius rate coefficients are $k_{1}, k_{2}$ and $k_{3}$ for the first, second and third reaction, respectively and have the following form:

$$
\begin{gathered}
k_{1}=10^{12} \exp (-30 / R T)\left[C_{3} H_{8}\right]^{0.1}\left[O_{2}\right]^{1.65} \\
k_{2}=10^{14.6} \exp (-40 / R T)[C O]\left[H_{2} O\right]^{0.5}\left[O_{2}\right]^{0.25} \\
k_{3}=5 * 10^{8} \exp (-40 / R T)\left[C O_{2}\right]
\end{gathered}
$$

resulting in the following reaction rates:

$$
\begin{gathered}
\dot{w}_{1, k i n}=k_{1}\left[C_{3} H_{8}\right]\left[O_{2}\right]^{3.5} \\
\dot{w}_{2, k i n}=k_{2}[C O]\left[O_{2}\right]^{0.5} \\
\dot{w}_{3, k i n}=k_{3}\left[\mathrm{CO}_{2}\right]
\end{gathered}
$$

Chemical reactions are mainly controlled by molecular species mixing but in the LES implementation a filtered reaction rate has to be included in the formulation. The subgrid linear-eddy mixing (LEM) model being integrated will address this smallscale reaction diffusion processes more accurately and the results using LEM within LES will be reported in the next quarter. Here, we incorporate a subgrid Eddy Break-Up (EBU) model for reaction rate closure for LES.

In the EBU model, the filtered reaction rate in the LES species equations are determined by comparing the time-scales for chemical reactions with the turbulent mixing time and then using the largest of the two. In the EBU model, the time required to complete molecular mixing is assumed to be of the same order of magnitude at the time required for a subgrid eddy to dissipate (i.e. momentum, species and energy diffusion and molecular mixing at the subgrid scale have the same time 
scale). The Lewis and Prandtl numbers are assumed to be unity at the subgrid scale in this formulation. More precisely, the chemical rate will be the minimum of the kinetic rate and the mixing rate. This means that the minimum rate is controlling the reaction process. The rate of dissipation of a subgrid eddy can be deduced from the subgrid turbulent kinetic energy and the subgrid dissipation. We then have the following relation:

$$
\dot{w}_{\text {mix }} \sim \frac{\epsilon^{s g s}}{k^{s g s}} \sim \frac{\left(k^{s g s}\right)^{\frac{1}{2}}}{l^{s g s}}
$$

where sgs denotes the subgrid scale, and $l^{s g s}=\Delta$ is the characteristic length of the cell. The subgrid kinetic energy $k^{s g s}$ is obtained from the SGS closure.

\section{Test Conditions for the DOE-HAT Combustor}

The diameter of the inlet and the combustor are identical to the DOE-HAT case and the length of the combustion chamber is $\sim 26.0 \mathrm{~cm}(11.0 \mathrm{in})$. This length is smaller than the actual test device but is chosen for the initial tests to verify the overlapping grid and LES implementation issues. The inflow characteristics chosen for the present study are: temperature of $699 \mathrm{~K}(800 \mathrm{~F})$, a inflow pressure of $19.78 \mathrm{~atm}$. (280 psi), a mean inflow velocity of $68.6 \mathrm{~m} . \mathrm{s}^{-1}$ and an equivalence ratio of 0.58 giving a mass flow rate of $0.986 \mathrm{~kg} . \mathrm{s}^{-1}$. The inflow velocity profile used was obtained from RANS data provided by the Pratt and Whitney Company. A random turbulent field in added to the inflow mean velocity and a subgrid turbulence intensity of around 5 percent is used to initialize the subgrid kinetic energy. As noted below, we are also developing a more comprehensive approach to generate realistic inflow turbulence for this configuration.

\section{Results and Discussion}

\subsection{Validation of the Overlapping Grid Methodology}

A major effort was expended in the last quarter to validate the accuracy of the overlapping grid for LES applications. Detailed evaluation has shown that this new approach is accurate and computationally very efficient in the parallel code. In addition to validation using analytical (exact) solutions, we have validated this approach using dump combustor flows. For example, a side view of an axial velocity field is shown in Fig. 3 and a front view of the radial and azimuthal component of the velocity vector in the centerline region are shown in Fig. 4. Figure 3 shows that the transition from one grid to the other does not introduce a jump in the isolines and the flow field is smooth with no discontinuities. Figure 4 shows similar continuous

features in the velocity field in the other directions. With these studies we believe the new LES solver is operational for DOE-HAT combustor flows. 
As mentioned earlier, a fourth and fifth-order interpolation methods are also being investigated for the fourth-order LES applications. The basic model remains unchanged and only the interpolation algorithm needs to be changed. We expect this version to be validated in the very near future.

\subsection{Thin-Flame LES of DOE-HAT Combustor Flow}

The DOE-HAT combustor geometry stabilizes the premixed flame by a combination of inlet swirl and the two dump planes at the entrance of the combustor. The sudden expansion imposes an adverse pressure gradient on the incoming flow and a recirculating bubble is formed downstream of the base of the inlet dump. This recirculation stabilizes the flame at a location upstream of the bubble. This location is determined by a balance between the adverse pressure gradient force and the forward momentum of the flow. In general, to form the recirculating bubble (often called the vortex breakdown phenomenon) in a sudden expansion dump combustor, the swirl intensity has to exceed a critical value. However, in the DOE-HAT combustor, the centerbody also provides another constraint to the flow expansion in the combustor and therefore, this geometrical features enhances the formation of the recirculation region even at lower values of swirl.

Most of these features can be resolved accurately with the G-equation thin flame model. Simulations using this model have been completed and analyzed to understand the flow features. Some characteristic results are report below.

An instantaneous view of the recirculation region predicted by the LES is shown on Fig. 5 and corresponds to the region inside the blue ellipse. However, in general, these regions are highly unsteady and further downstream a flapping motion in the base wake flow is observed. Figures 6 and 7 show the azimuthal vorticity iso-surfaces of the flow behind the bluff body. As can be seen, the vortical structures show both a swirling motion and a flapping motion (observed when multiple images are visualized). Initially, the vortical flow is highly coherent but further downstream, it break down into small-scale three-dimensional structures characteristics of $3 \mathrm{D}$ turbulence. The flapping or or motion normal to the streamwise flow can be seen more clearly in Figs. 8 and 9 where it can be observed that there is significant asymmetric motion in the flow. This phenomenon is related to the von Karman vortex shedding process from the corners of the center bluff body which combines with the flow in the wake behind the bluff body. Asymmetric vortex train is observed which is related to the corkscrew vortical field shown earlier.

A typical (time-averaged) $G=0.5$ iso-surface is presented on figure 10 . The average length of the flame is $\sim 2.5 \mathrm{~cm}$. The flame is anchored at the lip of the dump combustor and the base bluff body. Since, at present, the inlet has not be included, the interaction between the inlet flow and the dump combustor is not included. However, in the future we plan to include the inlet as well to study if the flame stabilization mechanism is modified by the presence of the inlet. 


\subsection{Finite-rate LES of DOE-HAT Combustor}

Simulations of the DOE-HAT flow is also underway using the finite-rate kinetics model combined with the EBU approach to estimate the filtered reaction rate term. Some preliminary results are report here.

We are simulating propane/air mixture with an equivalence ratio of 0.58 . Although the simulation is not completed, results show that the EBU based LES of this case is working as expected.

Figure 11 shows an iso-surface of the azimuthal vorticity. The helicoidal structure we see is caused by the flapping of the flow and is consistent with the observations in the thin-flame model. The temperature iso-surface $(\mathrm{T}=2000 \mathrm{~K})$ shown in Fig. 12 shows the location of the peak temperature region. This region is approximately the flame location (but not the exact location since the product region can also be at this temperature for some time downstream of the peak location. Therefore, to determine the actual location of the flame we need to used the propane reaction rate profile. This data is currently being acquired and will be reported soon. One interesting observation is that the flame surface is more 3D rather than the axisymmetric shape observed in the G-equation LES. However, the time-averaged data needs to be analyzed before any further conclusions can be made.

Another point to note that the eddy break model does not take into account local flame extinction due to high strain. Past studies have suggested that in regions of high strain, the EBU model will switch from the mixing time to the chemical time and predict a reacting process. However, experiments suggest that as the strain rate increases, local flame extinction can take place which can also impact the flame shape and location. An approach have been developed earlier to account for local flame extinction which restricts combustion in regions where the local strain rate exceeds the extinction value. This approach is very easy to implement and quite effective. Therefore, this model will be implemented in the near future.

Since we are using the two-step mechanism with CO chemistry, we can look at $\mathrm{CO}$ emission as shown in Fig. 13. As expected, a significant amount of $\mathrm{CO}$ is formed in the flame region although further downstream some recombination to $\mathrm{CO}_{2}$ and breakdown of $\mathrm{CO}_{2}$ into $\mathrm{CO}$ can also occur. The $\mathrm{CO}$ distribution is highly $3 \mathrm{D}$ as seen in 14 , which is a front view of the $\mathrm{CO}$ mass fraction.

\section{Conclusion and future plans}

A new overlapping grid LES method has been developed to study highly 3D flows as generated by the DOE-HAT combustor. Preliminary thin-flame LES studies have been completed and LES with finite-rate kinetics (using a subgrid EBU model) are now underway. The chemical model includes CO-chemistry in order to evaluate the effect of equivalence ratio on $\mathrm{CO}$ emission.

The next phase of this study will complete these EBU LES for three different equivalence ratio to determine if the variation in $\mathrm{CO}$ observed in the experiments can be captured in these simulations. For example, experiments show that as the 
equivalence ratio is decreased the $\mathrm{CO}$ emission first decreases and then suddenly increases. This phenomenon is expected to be related to the interaction between the flame and turbulent flow leading to local extinction and flame oscillations. Note that the $\mathrm{CO}$ trend with equivalence ratio has also been observed in other combustors (including smaller combustors at General Electric). Therefore, the trend should be captured in these LES if the chemistry model is reasonable. We plan to first see if the subgrid EBU approach can capture this phenomenon. If so, it may offer a relatively cheap approach to predict CO emission trends in realistic combustor. Since the LES with EBU model is operational, the completion of the study depends primarily on the turn-around time on the DOD HPC computers.

If these LES using subgrid EBU model shows an ability to predict the CO trend in this combustor (which is $26 \mathrm{~cm}$ long) we plan to continue this study using the much longer combustor $(40+\mathrm{cm})$ used in the actual DOE-HAT experiments. In this case, we will augment the current kinetics model with NO kinetics as well.

The next phase of this study will also begin integration of the online subgrid lineareddy mixing (LEM) model with more detailed kinetics using ISAT into the DOE-HAT problem. This approach is considered essential to evaluate the fundamental features that contribute to the observed CO emission trends, including flame-turbulence interactions and combustion instability. In general, the LEM with ISAT is already operational in our 3D LES code but its integration into the current DOE-HAT geometry will require careful re-evaluation due to the presence of the overlapping grid. However, we expect that this will not pose any major problems and we expect the integration to be completed within the next 1-2 months.

The LES with LEM+ISAT is expected to be very expensive computationally when detailed kinetics are employed and can only be used to understand the physics of the problem. To be able to carry out systematic design level studies, a more efficient approach needs to be implemented. This will involve the aforementioned ANN approach which will replace the entire LEM-ISAT model using a turbulent ANN. Some preliminary results of the integration of both the laminar and turbulent ANN have been obtained using a 3D LES model for a freely propagating flame under another study. Once this study is completed we plan to integrate the turbulent ANN into this code.

\section{References}

[Kim and Menon, 2000] Kim, W.-W. and Menon, S. (2000). Numerical simulations of turbulent premixed flames in the thin-reaction-zones regime. Combustion Science and Technology, 160:119-150.

[Kim et al., 1999] Kim, W.-W., Menon, S., and Mongia, H. C. (1999). Large-eddy simulation of a gas turbine combustor flow. Combustion Science and Technology, 143:25-62.

[Lilley, 1997] Lilley, D. (1997). Swirl flows in combustion: A review. AIAA Journal, 15(8):1063-1078. 
[Peters, 2000] Peters, N. (2000). Turbulent Combustion. Cambridge Monographs on Mechanics. 


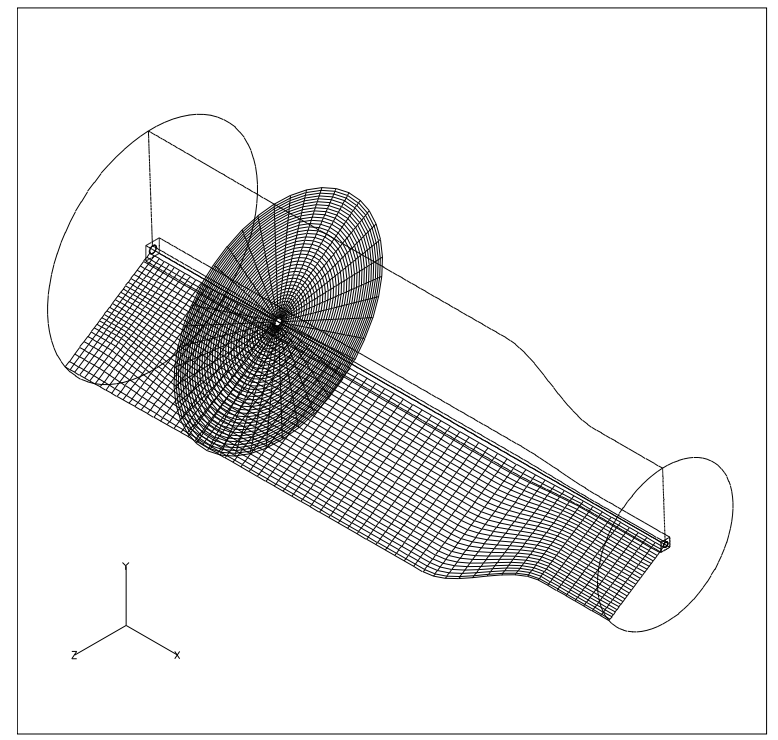

Figure 1: 3D view of the new overlapping grid mesh

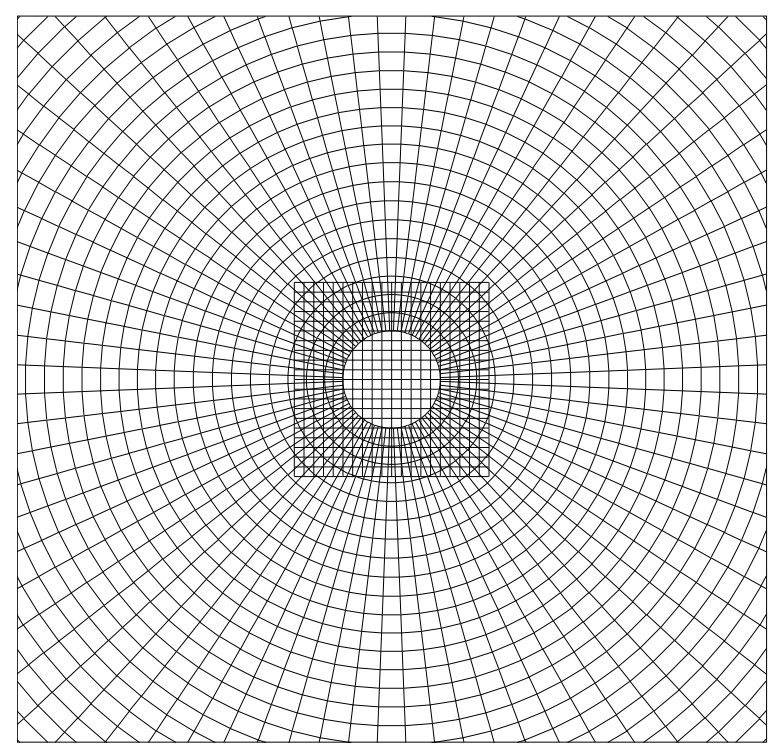

Figure 2: Front (zoom) view of the grid mesh showing the two overlapping grids 


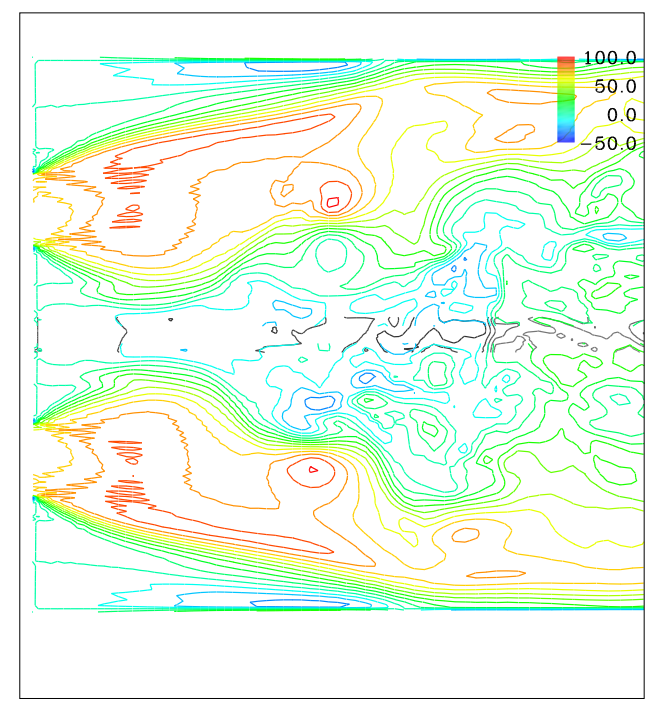

Figure 3: Instantaneous axial velocity field from both grids. Color contours: data from the cylindrical grid, Gray scale contours: data from the cartesian grid. As can be seen there is no discontinuity between the two fields

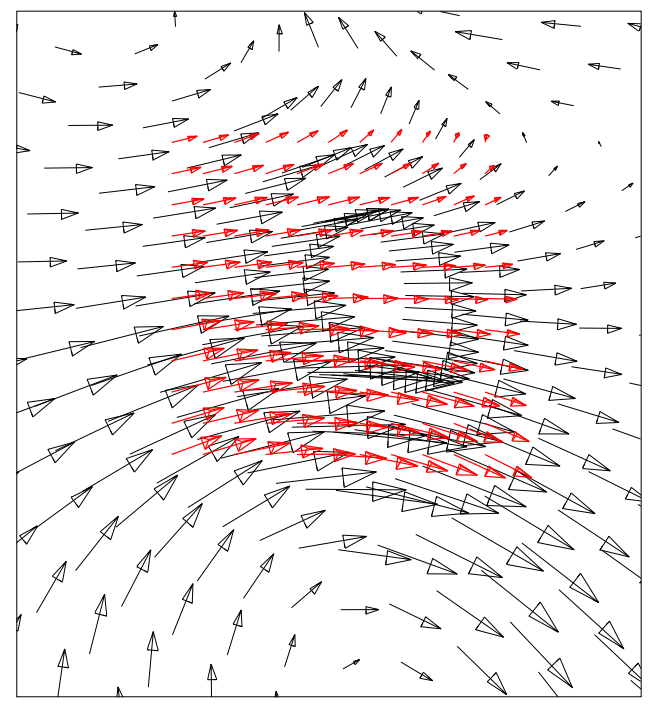

Figure 4: Front view of the radial and azimuthal components of the velocity vector in the center line region. Black arrows: data from the cylindrical grid, red arrows: data from the cartesian grids. 


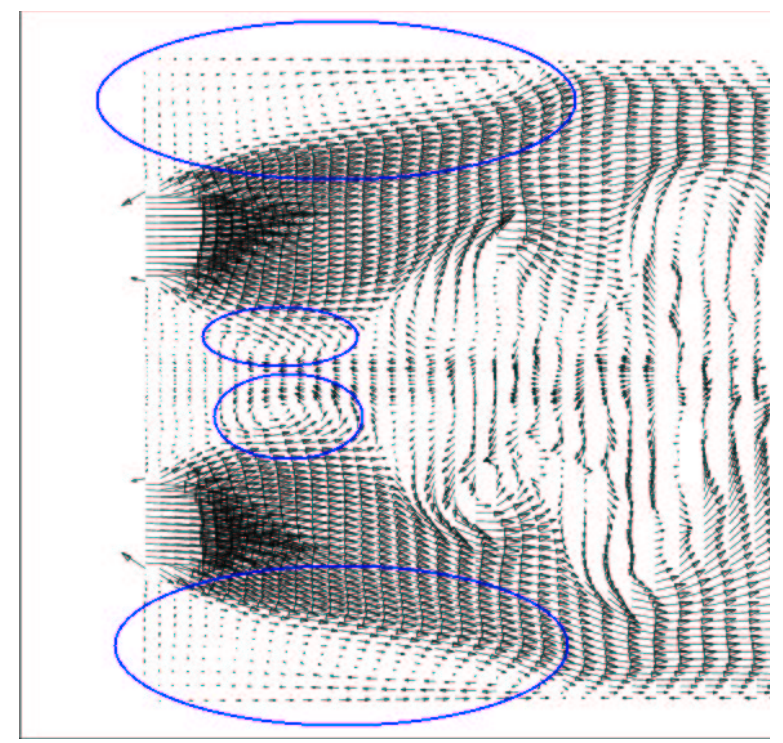

Figure 5: Velocity vector field in the DOE-HAT combustor. Note that the blue ellipses show the region where recirculating flow was observed.

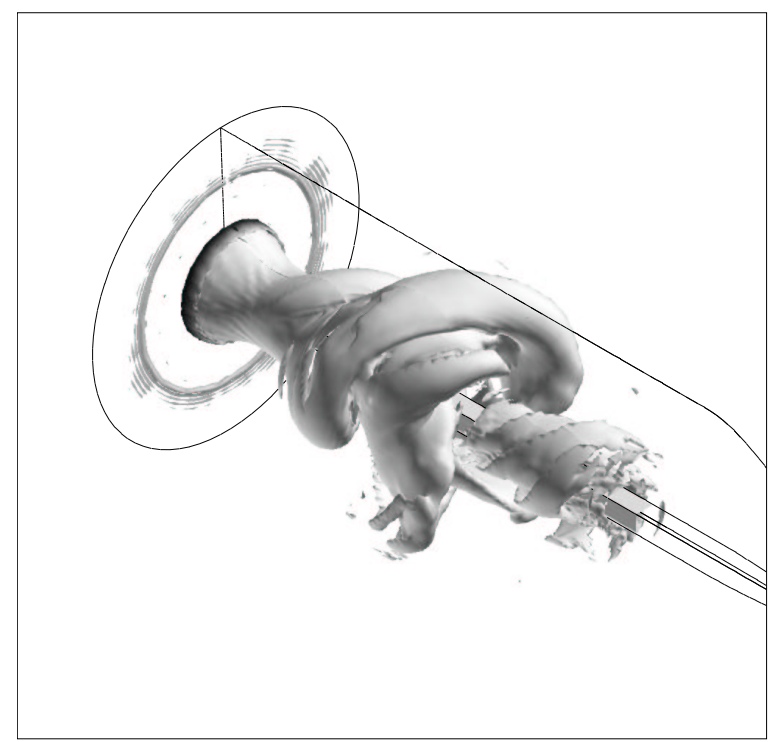

Figure 6: Azimuthal vorticity Iso-surface $\left(5500 \mathrm{~s}^{-1}\right)$ showing the swirling wake behind the center body. 


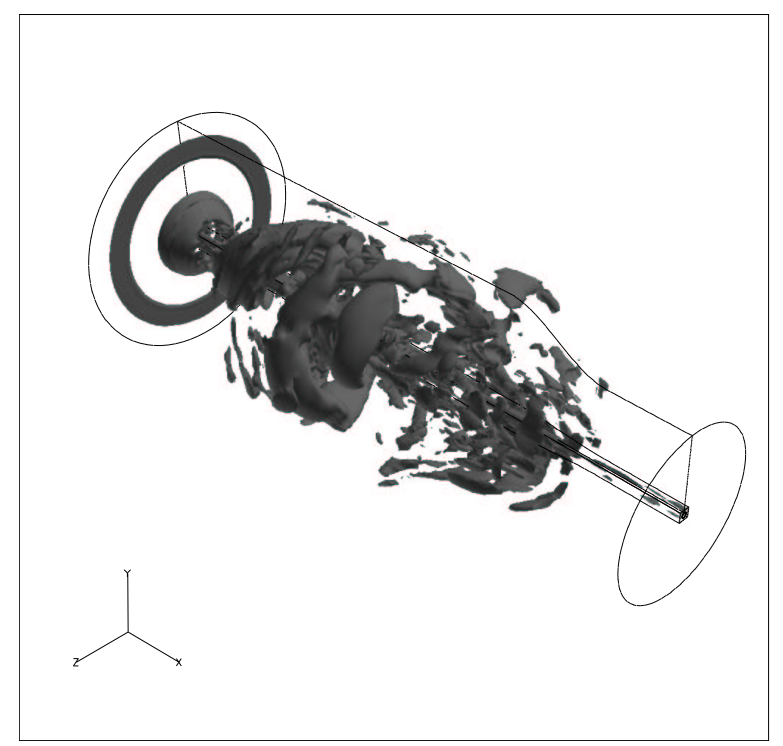

Figure 7: Azimuthal vorticity Iso-surface $\left(6500 \mathrm{~s}^{-1}\right)$. At this higher value, the presence of smaller structures formed during vortex breakdown can be seen.

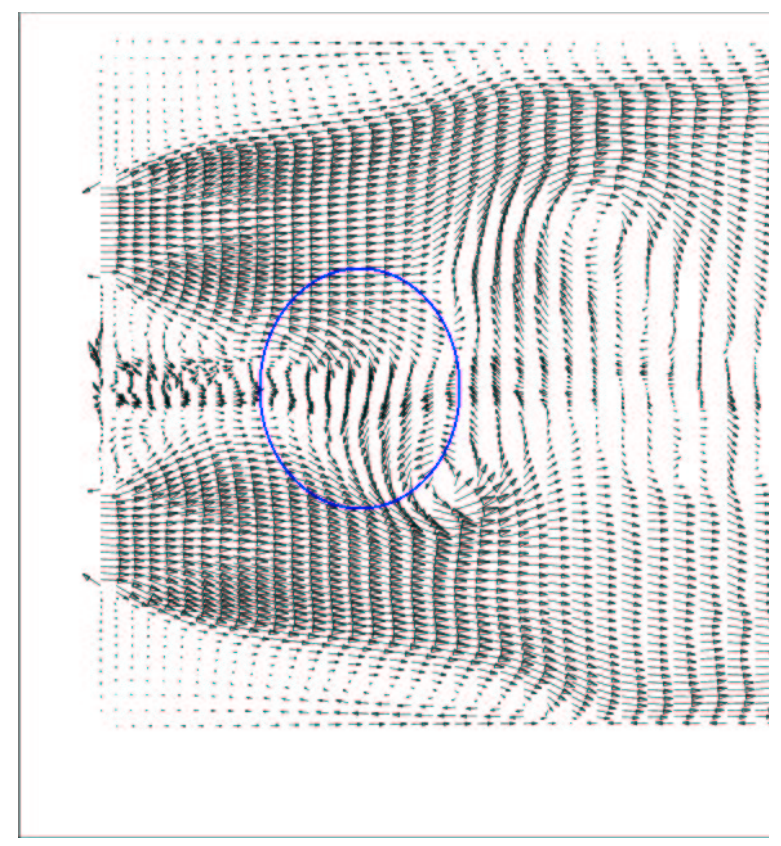

Figure 8: Velocity vector field with the region where wake flapping due to asymmetric vortex motion is observed. The blue ellipse marks the approximate location where flapping is observed. 


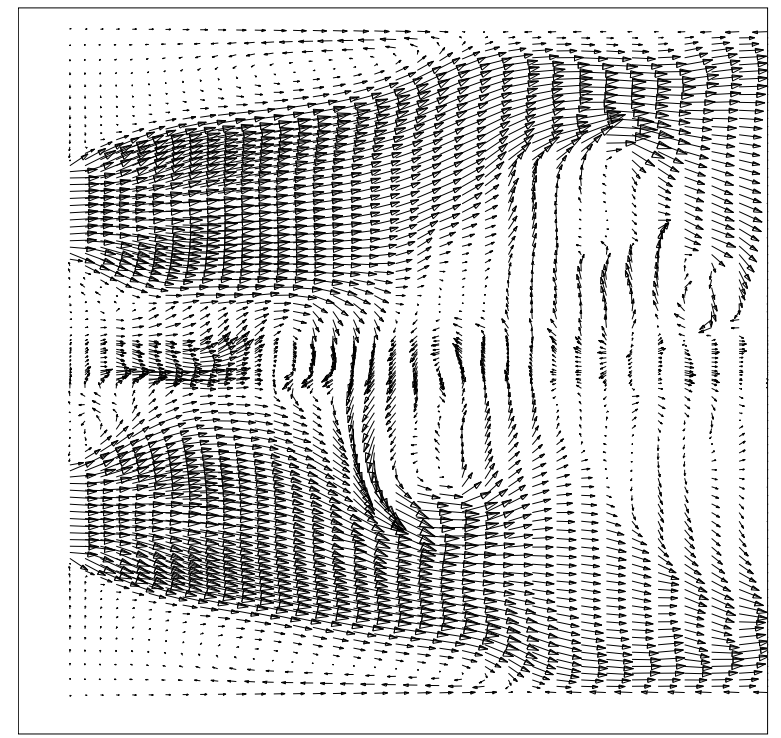

Figure 9: Velocity vector field showing another instant as above showing the formation of asymmetric vortex formation and motion in the wake.

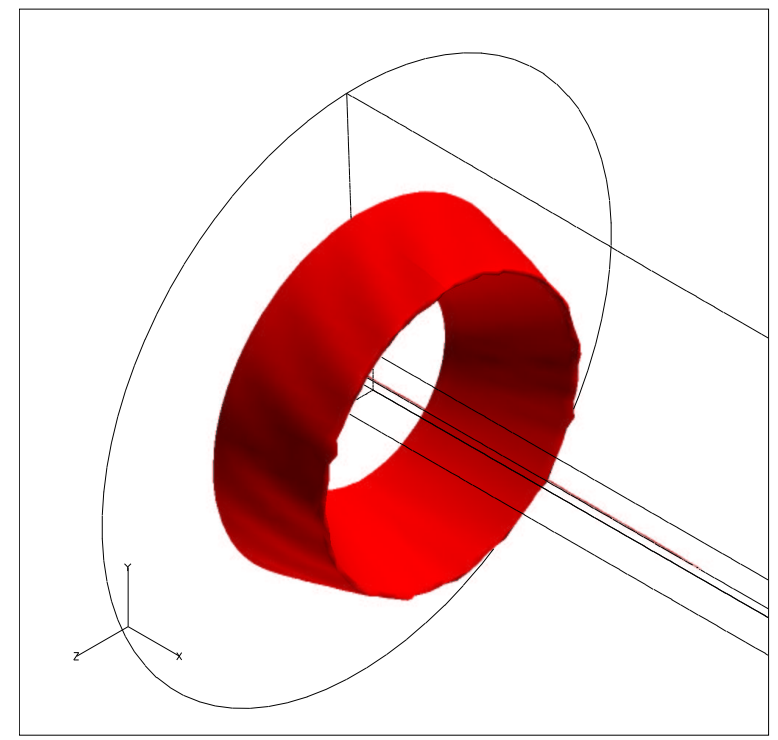

Figure 10: The time-averaged thin flame surface identified with the $\mathrm{G}=0.5$ Isosurface. Notice that the flame is anchored at the lip of the dump plane and the center body. 


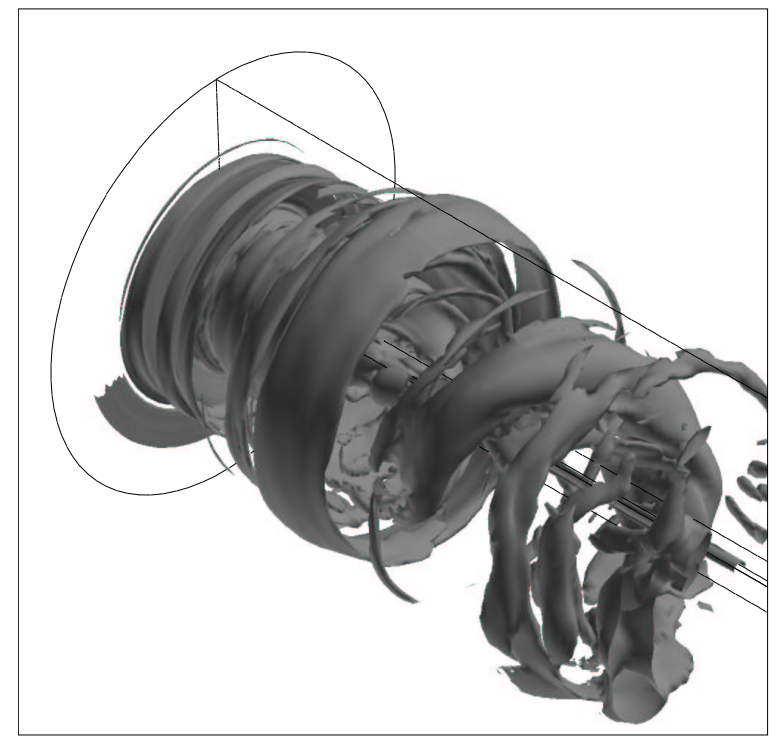

Figure 11: Azimuthal vorticity iso-surface $\left(\mathrm{w}=6500 s^{-1}\right)$ as predicted by the LES using the finite-rate LES model. The general shape and structure of the flow in the wake of th center body is similar to that seen in the earlier thin-flame LES.

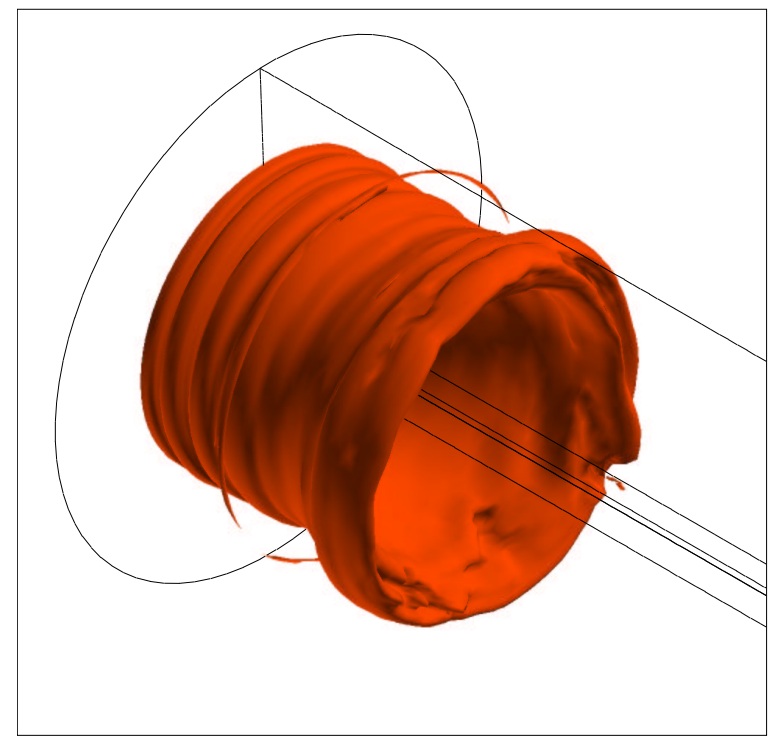

Figure 12: The temperature iso-contour for $\mathrm{T}=2000 \mathrm{~K}$ as predicted by the LES using finite-rate kinetics with the subgrid EBU model. 


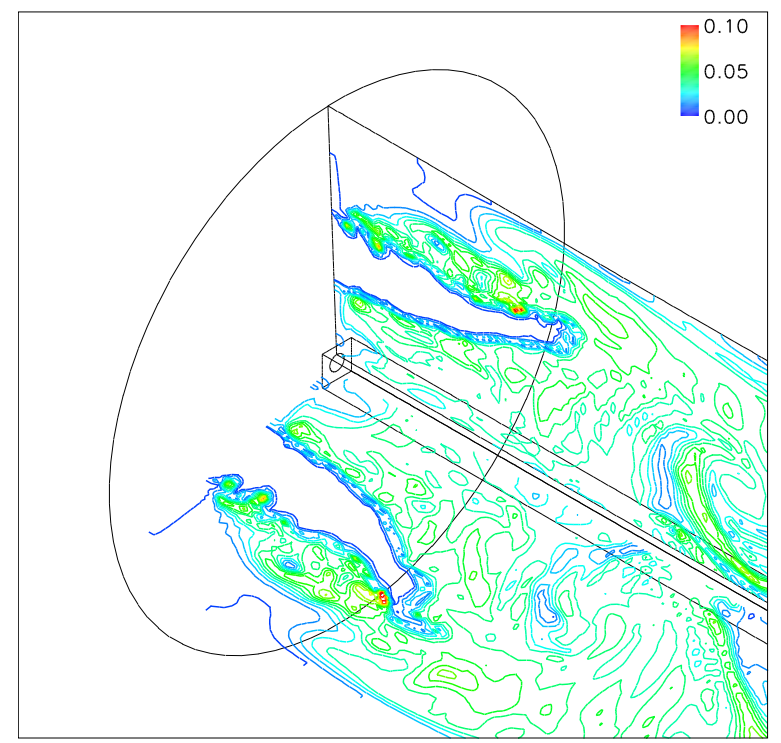

Figure 13: The CO mass fraction field in the combustor shown on two planes. Significant 3D structure appears in these views with majority of CO forming in the flame zone.

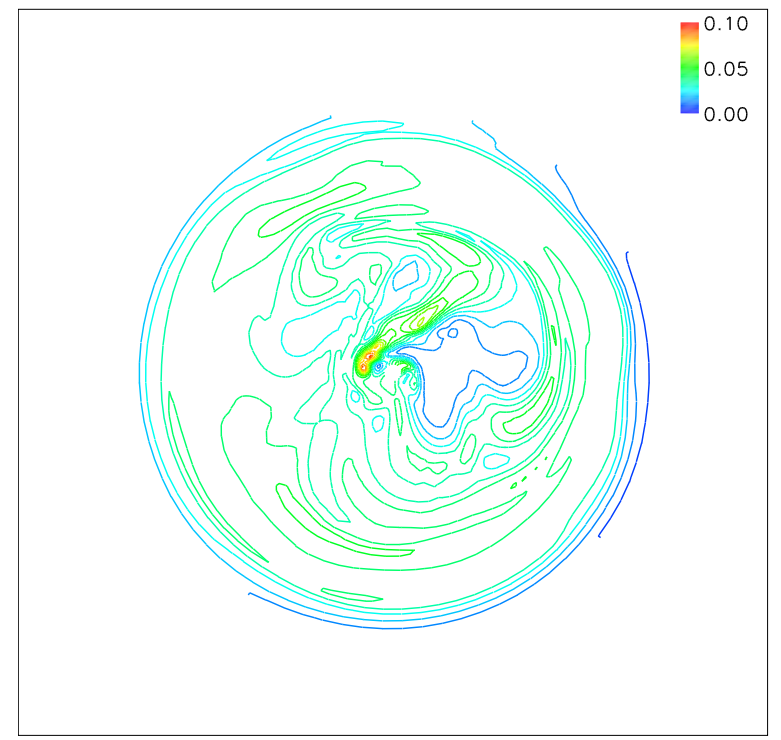

Figure 14: Front view of the CO mass fraction field showing the asymmetric shape of the CO field. 